\title{
Energy concentration at the centre of large aspect ratio rectangular waveguides at high frequencies
}

\author{
F.B. Cegla \\ February 21, 2008 \\ Department of Mechanical Engineering \\ Imperial College London \\ London SW7 2AZ \\ United Kingdom
}




\section{ABSTRACT}

Waveguides in NDE applications are commonly of a regular geometry (e.g. circular, ring cross section) for which analytical solutions exist. In this paper wave propagation in infinitely long strips of large rectangular aspect ratio is discussed. Due to the finite width of strips a large number of modes exist within the structure. This complicates the analysis and usually discourages the use of strip-waveguides in NDE sensors. However it is shown that among the many modes of a strip there are some with very desirable properties. This is highlighted by the example of two guided wave modes of a large aspect ratio rectangular strip whose dispersion characteristics approach those of the fundamental modes of an infinitely wide plate at high frequencies. The energy of these modes concentrates in the central region of the strip and decays towards the edges so that the strip waveguide can easily be mechanically attached to other components without influencing the wave propagation. Dispersion curves and mode shapes were derived using a Semi Analytical Finite Element (SAFE) technique and are presented over a range of frequencies. It is shown that selective excitation of both modes is possible in practice and the experimental setup is described.

PACS numbers: 43.20.Mv, 43.40.Le 


\section{INTRODUCTION}

The analysis of guided wave propagation in infinitely wide plates, rods and pipes is well established ([1], [2], [3], ...) and has been made use of in numerous NDE applications such as large area inspection (eg. [4], [5]), long range pipe inspection (eg. [6]) and material property measurements (eg. [7], [8]) to mention only a few. Applications often make use of the low frequency regime, where the modal density is low and selective mode excitation is easily possible. Areas of high modal density such as at high frequency are usually avoided because of the need for more sophisticated transducers and narrower bandwidth of operation. In plates of finite width (strips) the modal density is high due to additional modes in the width direction. The conventional wisdom would suggest to avoid these geometries since excitation of selected modes would be more difficult. However in certain applications a strip geometry can offer an advantage over other geometries.

The author was interested in the use of waveguides to convey ultrasonic signals from a remote transducer to a component that is to be investigated. Literature on source characteristics of sources of different geometry and surface loading on half-spaces [9], [10] showed that anti-plane shear line sources are potentially an attractive way to maximise transmission into half-spaces. Since line sources do not exist in real life, large aspect ratio rectangular sources (width $>>$ thickness) are the closest practically implementable approximation. To transmit surface loads that are applied over a rectangular cross section onto components the author analysed wave propagation in in- 
finitely long strip waveguides of large rectangular aspect ratio. During the analysis the attention was focused on two modes that resemble the $\mathrm{SH}$ and A0 modes of a plate of infinite width at high frequencies. Their properties and experimental excitation are described in the following sections.

\section{THEORY - WAVE PROPAGATION IN RECTANGULAR STRIPS AND THEIR DISPERSION CHARACTERISTICS}

The modelling of wave propagation remote from the edges in very wide plates is accurately achieved by modelling plane wave propagation under plane strain conditions. Software packages that accurately model the wave propagation in plates under these conditions (e.g. DISPERSE [11]) exist. These models work well in cases where the width (W) of the plate and propagation distance (L) are comparable and much larger than the thickness $(\mathrm{T})$. As the propagation distance increases to be larger than the plate width, reflections from the sides start to appear in the received signals and it becomes necessary to deal with the three dimensional problem. This can be considered as the propagation along a rectangular strip with constant cross section.

Mindlin and Fox [12] were the first to describe the propagating modes of a bar of rectangular cross section. Their solution was made up of a superposition of the flexural, longitudinal and shear modes that propagate in infinitely wide plates of 2 different thicknesses. The thickness of the plates 
corresponded to the width and thickness of the rectangular bar. The solutions for the infinitely wide plates were rotated by $90^{\circ}$ relative to each other and superposed in order to fulfil the boundary conditions of zero stress all around the perimeter of the cross section. This method enabled them to determine the propagating modes of the bar at distinct frequencies and aspect ratios of the bar, but a solution for all frequencies and aspect ratios was not possible. Also Fraser [13] presented an analytical method to calculate the dispersion curves for infinitely long rectangular bars accurately for a limited range of wavenumbers. More recently the continuous tracing of dispersion curves for wave propagation in structures of arbitrary cross section has become possible through the use of finite element (FE) eigensolvers. Wilcox et al. [14], Mukdadi et al. [15], Hayashi et al. [16], Gavric [17], [18] and others have reported methods of tracing dispersion curves for a range of different sections (L-shaped sections, rail heads and rectangular strips). These techniques are now widely used and are often referred to as the SAFE (Semi Analytical Finite Element) method. Predoi et al. [19] give an overview and a number of references about the development of finite element techniques for modelling of wave propagation in waveguides.

The method of Wilcox et al.[14] has been employed here to analyse the modes propagating in $1 \mathrm{~mm}$ thick steel strips of a much larger width (> $15 \mathrm{~mm})$. The method works by defining an axisymmetric model with a very large radius compared to the dimensions of the cross section. The section of the axisymmetric body represents the cross section of the wave guide, see figure 1. Due to the very large radius, the structure approximates a straight 
waveguide. For the finite element eigensolver a specific cyclic order can be specified. This specifies the number of wavelengths that exist around the circumference of the axisymmetric body. For example a cyclic order of 1 corresponds to a wavelength equal to the circumference of the structure. For a cyclic order of 2 there are two wavelengths around the circumference and so on. Therefore the wavelength of the solution is determined by the following equation:

$$
\lambda=\frac{2 \pi R}{C_{\text {order }}}
$$

where $\mathrm{R}$ is the radius of the model and $C_{\text {order }}$ is the cyclic order of the FE eigensolver. At each cyclic order the FE-eigensolver routine will determine several resonance frequencies, each frequency corresponding to a different mode. The wavenumber dispersion curves for the meshed cross section can now be determined by plotting the resonance frequencies against the wavenumber, which is determined using equation (1) and the identity

$$
k=\frac{2 \pi}{\lambda}
$$

where $\mathrm{k}$ is the circular wavenumber and $\lambda$ the wavelength. This method therefore yields a set of discrete frequency solutions at each wavenumber. A typical set of results is shown in figure 2(a). Wilcox et al. [14] developed software that connects adjacent solution points to form a continuous solution line (mode) in the wavenumber frequency domain. The joining up of adjacent points to form a line is carried out by comparing the mode shapes of adjacent solutions and by using the slope of the curve of existing solutions to predict 
the continuation of the curve, which is similar to the method presented by Lowe [3]. The finite element software that determined the eigen solutions was the FINEL 77 code which was developed by Hitchings [20] at Imperial College.

Phase velocity dispersion curves can be obtained from the wavenumber frequency plot by converting them using the following identity:

$$
C_{p h}=\frac{\omega}{k}
$$

Once the phase velocity is determined the group velocity can be calculated using:

$$
C_{g r}=\frac{\partial \omega}{\partial k}=C_{p h}+k \frac{\partial C_{p h}}{\partial k}
$$

An interesting aspect to note about the technique is that the determined FE eigen solutions are confined to a rectangular domain in the frequency wavenumber space. However when this is transformed into a phase velocity frequency space the solutions will be bound in a space between the two lines $C_{p h}=\left(1 / k_{\min }\right) \omega$ and $C_{p h}=\left(1 / k_{\max }\right) \omega$. This is illustrated in figure $2(\mathrm{~b})$. The mode shapes of each mode are a direct result of the FE analysis and can be extracted at each frequency.

The technique described was used to generate dispersion curves for rectangular strips. The results for a steel $\left(\rho=7932 \mathrm{~kg} / \mathrm{m}^{3}, E=216.9 \mathrm{GPa}\right.$, $\nu=0.2865$, unless otherwise stated) strip geometry of $1 \mathrm{~mm}$ thickness and 
width $30 \mathrm{~mm}$ are presented here. The radius of curvature of the FE model was $2 \mathrm{~m}$ in order to ensure accurate results. Wilcox et al. [14] reported the appearance of discrepancies of the solution to the curved model and the analytical solution above frequencies of $4 \mathrm{MHz}$. Figure $3 \mathrm{a}$ and $\mathrm{b}$ shows the frequency-wavenumber and phase velocity-frequency dispersion curves. For comparison, figure 3c and d shows the phase velocity dispersion curves for a $1 \mathrm{~mm}$ thick infinite plate (c) and a $30 \mathrm{~mm}$ thick infinite plate (d) of the same material. The DISPERSE software [11] was used to trace the curves in figure $3 \mathrm{c}$ and $\mathrm{d}$. Many more modes with phase velocities below $3 \mathrm{~m} / \mathrm{ms}$ exist at low frequencies in the strip than in the infinitely wide plate case; these extra modes are due to the finite width of the strip.

\section{ANALYSIS OF THE SH0* AND A0* MODE}

Two modes are highlighted in figure 3 ; These modes were named $\mathrm{A} 0^{*}$ and SH0* (the * here and for the remainder of the paper indicates a strip mode), after the well known fundamental A0 and SH0 plate modes because their high frequency dispersion characteristics tend towards those of these plate modes (assuming the smallest dimension to be the thickness). In addition to this their mode shapes in the high frequency limit also are of the same polarisation as the fundamental A0 and SH0 plate modes however they are concentrate at the centre of the strip and their amplitudes decay towards the edges of the strip. (The naming of these two strip modes thus does not follow the same rules as the conventional naming of Lamb wave or SH wave modes 
but is done by comparing their properties and adding the ${ }^{*}$ to indicate that it is a strip mode). The $\mathrm{A}^{*}$ and $\mathrm{SH} 0^{*}$ modes are now investigated in detail.

\section{A The $\mathrm{SH}_{0} *$ Mode}

Strictly speaking the term 'shear horizontal' does not make sense in a geometry other than an infinite plate. Therefore it is stressed here again that the name $\mathrm{SH}^{*}$ mode was chosen due to the high frequency characteristics of the mode. At high frequencies the $\mathrm{SHO}^{*}$ mode travels with the shear velocity of the material and exhibits strong displacements in the y-direction only that decay from the strip centre towards the edges (the behaviour on the centre line being the same as that of the SH0 mode in an infinite plate). The same mode could also be described as a bending mode of the strip in the width-propagation direction (y-z) plane being similar to the A1 Lamb mode in an infinitely wide plate of thickness equal to the width of the strip. Just like the A1 Lamb mode the SH0* modes possess a cut off frequency below which it does not propagated. Due to the finite strip width the SH0* mode wavenumber in the propagation direction becomes imaginary below the cut off frequency and the field decays exponentially along the waveguide axis.

The phase velocity dispersion curves for the SH0* mode of a $1 \mathrm{~mm}$ thick steel strip of different widths $(30,15$ and $7.5 \mathrm{~mm})$ are shown in figure $4(\mathrm{a})$. The SH0* mode has a cut-off that depends on the width of the strip. At frequencies well above the cut-off, the phase velocity asymptotically approaches the bulk shear velocity of the strip material. Figure 4 b shows that the disper- 
sion behaviour is a function of the frequency-width product, all three curves of figure $4 \mathrm{~b}$ being coincident.

While strongly frequency (or width) dependent at low frequency-width products, at high frequency-width products the $\mathrm{SH} 0^{*}$ mode phase velocity is constant and equal to the bulk shear velocity, allowing non-dispersive wave propagation without signal distortion. The transition from highly dispersive to non-dispersive is virtually complete at a frequency-width product of $15 \mathrm{MHz}-\mathrm{mm}$ where the $\mathrm{SH}^{*}$ mode velocity differs by less than $1 \%$ from the bulk shear velocity. This point is marked by a vertical line in figure $4 \mathrm{~b}$ and marks the frequency-width product at which the wave-propagation is becoming non-dispersive for most practical purposes. The $15 \mathrm{MHz}-\mathrm{mm}$ criterion above which $\mathrm{SH}^{*}$ propagation becomes non-dispersive is material property dependent and is specific to steel that was used in the analysis here. To extend the criterion to other materials it is useful to specify a minimum strip width in terms of shear bulk wavelengths. $15 \mathrm{~mm}$ equates to roughly 5 bulk shear wavelengths at $1 \mathrm{MHz}$ in steel. As a rule of thumb it can therefore be expected that the strip width has to be larger than 5 shear bulk wavelengths of the waveguide material in order to permit non-dispersive wave propagation in form of the $\mathrm{SH}^{*}$ mode.

The mode shapes of the $\mathrm{SH}^{*}$ mode at different frequencies are shown in figure 5. The figure shows that at high frequencies the y displacement component is dominant and concentrated in the centre of the strip. The mode shape is constant across the thickness (x direction). Near cut-off (55kHz for 
$30 \mathrm{~mm}$ wide strip) there are displacements at the edges of the strip which diminish as the frequency increases. This is better illustrated in figure 6 which shows the evolution of the dominant y displacement across the width of the strip over a range of frequencies. In the graph each line represents a mode shape starting from $200 \mathrm{kHz}$ and increasing in steps of $100 \mathrm{kHz}$ up to $3 \mathrm{MHz}$. At a frequency-width product of $15 \mathrm{MHz}-\mathrm{mm}$ (i.e. the 4 th line in figure 6) the mode shape has started to concentrate in the centre of the strip, displacements at the edge have decayed and the shape has become similar to the final high frequency parabolic profile. A further increase in frequency will only slightly refine the mode shape towards its final shape.

If the mode is to be used in practice it is important to be able to excite it in a reliable way. It was investigated how accurately an exciting transducer would have to reproduce the mode shape in order to selectively excite the SH0* mode. The influence of the distribution of the excitation force was investigated using a finite element model. A finite element model for a $15 \mathrm{~mm}$ wide steel strip was prepared in the ABAQUS finite element software [21]. The model was two dimensional with a plane stress condition in the thickness direction of the strip. Due to its special polarisation the SH0* mode only contains $\sigma_{z y}$ stress components, which satisfy the plane stress condition $\left(\sigma_{x x}=\sigma_{x y}=\sigma_{x z}=0\right)$. A frequency domain solver was used. At one end of the strip a force was applied while an absorbing region at the other end of the strip prevented any reflections of the excited waves. This technique is commonly used to remove the influence of unwanted reflections from boundaries in FE models [22], [23]. 
A sketch of the FE model is displayed in figure 7. Square quadratic elements of size $0.25 \mathrm{~mm}$ were used to mesh the strip and the absorbing region. The viscoelastic parameters of the absorbing region were increased in a cubic fashion from the interface with the strip. They were determined as described by Drodz et al. [23]. Different distributions of exciting force over the width of the steel strip $\left(\rho=7932 \mathrm{~kg} / \mathrm{m}^{3}, E=216.9 \mathrm{GPa}, \nu=0.2865\right)$ were used to see the influence of the excitation force profile on the waves excited in the strip.

The results of the $\mathrm{FE}$ analysis are displayed in figure 8. In the figure three different profiles of excitation force across the width are shown, together with the y (width) direction displacement field that they produce; the excitation frequency was $2 \mathrm{MHz}$. In the picture the displacement fields are normalised to show displacements on a scale between +100 and -100 so that the relative amplitude of the modes excited by the different force profiles could be assessed. For a uniformly applied force the displacement field in the strip becomes relatively complicated. It can be concluded that many modes are excited and interfere. If the stress profile of the $\mathrm{SH} 0^{*}$ mode (from FE eigensolver) is applied at the strip end, a pure mode can be excited in the strip [24]. Displacements are concentrated at the centre of the strip. For a triangular forcing profile, the $\mathrm{SH}^{*}$ mode is also preferentially excited. There is a marginal difference in the displacement field excited by the exact mode shape forcing compared to the triangular forcing profile; the difference is only visible at the edges of the strip where the amplitude of the $\mathrm{SH} 0 *$ mode is 
weakest. This shows that other modes are excited at much lower levels than the SH0* mode and their contribution to the overall displacement field is negligible. It was concluded that any excitation that is constant across the thickness of the strip and resembles the mode shape better than a triangular forcing in the width direction will allow the excitation of an almost pure SH0* mode in the strip.

\section{B The A0* Mode}

The A0* mode is very similar to the commonly known A0 mode in an infinitely wide plate. It is a flexural mode with respect to the thickness (x direction); however due to the finite width the mode also has a variation across the width (y direction). Just as the dominant displacements (y-direction) of the SH0* mode are symmetric about the width, the dominant displacements (x-direction) of the $\mathrm{A} 0 *$ mode are also symmetric about the width with maximum displacement at the centre of the rectangular strip. The relationship of the mode to the A0 mode in an infinitely wide plate is underlined by their similarity in phase velocity. Figure 9 shows the phase velocity of both modes as a function of frequency, the difference being greatest at low frequency near the cut off of the A0* mode and becoming negligible at high frequency. This trend is similar to the $\mathrm{SH}^{*}$ mode case where the strip mode asymptotically approaches the plate mode properties at high frequency.

The mode shape of the $\mathrm{A}^{*}$ mode also shows similar behaviour to the SH0* mode as the dominant displacement concentrates in the centre of the 
strip with increasing frequency. Figure 10 shows the mode shape of the A0* mode of a $1 \mathrm{~mm}$ thick, $30 \mathrm{~mm}$ wide strip at different frequencies. The main displacement component is in the $\mathrm{x}$ direction and displacements are significantly concentrated at the centre of the strip as the frequency increases. This is better seen in figure 11 where the $\mathrm{x}$ displacement component on the centre line of the strip is shown over a range of frequencies.

So far displacement components have been shown to illustrate the polarisation of the mode as well as its concentration of energy at the centre. Being proportional to the square of the displacement amplitude, the concentration of the mode energy is even more drastic.

\section{EXPERIMENTS}

The experimental investigation of wave propagation in rectangular strips was focused on exciting $\mathrm{A}^{*}$ and $\mathrm{SH}^{*}$ modes. To achieve this the exciting transducer has to mimic the mode shape as closely as possible and, for broadband signals, the mode shape should not change significantly over the range of excited frequencies. As shown in figure 8 it is insufficient to simply impose an uniform excitation across the waveguide width. However, mode shapes being uniform across the thickness the transducer output has to be varied across the width of the strip only (see figures 5 and 10). Displacements for

both modes are strong at the centre of the strip and decay towards the edges in approximately parabolic fashion. The main difference between the two 
modes is the polarisation of the $\mathrm{A} 0^{*}$ mode in the thickness $(\mathrm{x})$ direction and the polarisation of the $\mathrm{SH}^{*}$ mode displacements in the width (y) direction.

It was found that good results could be achieved by simply coupling a standard circular ultrasonic shear transducer (Panametrics V154) to the end cross section of the strip. The circular shape of the piezo electric element within the transducer was believed to transmit larger shear stresses at the centre of the strip width than at the outside and thus lead to preferential excitation of the $\mathrm{SH}^{*}$ or $A 0^{*}$ mode when rotated by $90^{\circ}$.

\section{A SH0* Mode Excitation}

Figure 12 shows a 5 cycle $2 \mathrm{MHz}$ Hanning windowed toneburst that was sent and received in pulse echo mode from a $15 \mathrm{~mm}$ wide, $1 \mathrm{~mm}$ thick and $300 \mathrm{~mm}$ long stainless steel strip. The $\varnothing 13 \mathrm{~mm}$ transducer (Panametrics V154) was clamped to the steel strip by a purpose made clamp using treacle (a very viscous fluid, similar to honey) as a shear couplant between the transducer face and the waveguide end section. The transducer was polarised in the direction of the width of the strip (y direction). Figure 12 shows that a very clean signal without significant dispersion can be excited and received in the strip. The presence of other modes about $30 \mathrm{~dB}$ weaker than the main signal can also be seen in figure 12. The presence of higher order modes that are notably slower can be explained by transducer misalignment and other imperfections within the strip and during reflection at the waveguide end. 
To be certain that the desired mode was excited in the experiment an in-plane dual head laser doppler vibrometer (Polytech OFV 512) was used to measure the in-plane surface displacement (y direction) of the strip along the centre line of the strip. The signal was recorded every $0.5 \mathrm{~mm}$ over a distance of $200 \mathrm{~mm}$ at a sampling frequency of $10 \mathrm{MHz}$. This is schematically illustrated in figure 13(a). From the measurements a two dimensional Fourier Transform was computed [25]. The 2D-FFT displays the frequency- wavenumber relationship of the signals that have been measured in the waveguide. This plot can directly be compared to analytical frequency-wavenumber predictions. In figure $13(\mathrm{~b})$ the $2 \mathrm{D}-\mathrm{FFT}$ result for a $30 \mathrm{~mm}$ wide and $1 \mathrm{~mm}$ thick steel strip is plotted. A line indicating the theoretically predicted SH0* mode frequency-wavenumber relation for a $30 \mathrm{~mm}$ wide and $1 \mathrm{~mm}$ thick steel strip $\left(\rho=7932 \mathrm{~kg} / \mathrm{m}^{3}, \mathrm{Cl}=6000 \mathrm{~m} / \mathrm{s}, \mathrm{Cs}=3060 \mathrm{~m} / \mathrm{s}\right)$ is also displayed. There is very good agreement between the measured data and the predicted values for the $\mathrm{SH}^{*}$ mode. The noise floor in the 2DFFT plot is relatively high; this is due to strong noise and drop outs in the laser vibrometer measurements.

\section{B A0* Mode Excitation}

The setup was slightly changed to excite the $A 0^{*}$ mode. A $30 \mathrm{~mm}$ wide, $0.2 \mathrm{~mm}$ thick and $300 \mathrm{~mm}$ long steel strip was used and the transducer was turned by $90^{\circ}$ to excite displacements in the thickness (x) direction. Figure 14(a) shows the pulse echo signal received by the transducer following excita- 
tion by a 10 cycle Hanning windowed toneburst with $2 \mathrm{MHz}$ centre frequency. The $A 0^{*}$ mode is very dispersive in this frequency range which explains the very strong distortion of the signal. Despite the strong dispersion the signal is due to a single dominant mode as suggested by Figure 14(b) which shows a smooth spectrum of the signal without dips and interference from other modes.

Again to verify the excited modes in the strip the laser vibrometer was scanned along the centre line of the strip and a 2D-FFT was computed. Figure 15 shows the result. Out-of-plane (x direction) displacement measurements were carried out over a distance of $200 \mathrm{~mm}$ at increments of 0.5 mm with a temporal sampling frequency of $10 \mathrm{MHz}$. The 2D-FFT shows that the $A 0^{*}$ mode is the dominant mode. Other modes are $\sim 20 \mathrm{~dB}$ less strong than the $\mathrm{A} 0 *$ mode.

\section{CONCLUSIONS}

Two particular guided wave modes $\left(\mathrm{SH}^{*}\right.$ and $\left.\mathrm{A} 0^{*}\right)$ of a large aspect ratio rectangular cross section waveguide were investigated in detail. It is shown that in both modes energy concentration at the centre of the waveguide is observed at high frequencies. At the same time the mode characteristics converge towards those of the fundamental modes ( $\mathrm{SH}$ and $\mathrm{A} 0$ ) in the infinitely wide plate case. One of the modes also assymptotically approaches the shear velocity at high frequencies and therefore becomes almost non-dispersive, 
which allows the propagation of undistorted signals over large distances.

It is experimentally demonstrated that these modes can easily be excited by standard techniques with signal to coherent noise ratios of $20 \mathrm{~dB}$ and better. Essentially, at large frequency-width products the modes that concentrate at the strip centre resemble the fundamental plane strain guided wave modes of a plate in the central region of the strip and decay towards the edges of the strip. For the $\mathrm{SH}^{*}$ mode it was possible to define a minimum strip width above which wave propagation will be non-dispersive. Any SH0* signal whose wavelength is smaller than a fifth of the strip width will propagate virtually non-dispersively at the bulk shear velocity along the waveguide centre.

Potential applications of these modes are in any field where guided plate waves are to be used but space is confined. An example from the author's experience is the development of non-dispersive buffer waveguide strips for high temperature thickness gauging. The waveguide allows the separation of the transducer from the measurement zone which can be useful for transducer longevity and accessibility reasons. Work on the development of the thickness gauge will be presented in a future publication. 


\section{ACKNOWLEDGEMENTS}

The author would like to thank Prof. P. Cawley for encouragement and the continued discussions on the topic of this paper.

\section{References}

[1] K. F. Graff. Wave Motion in Elastic Solids. Dover Publications inc., New York, 1973.

[2] J. L. Rose. Ultrasonic Waves in Solid Media. Cambridge University Press, 1999.

[3] M. Lowe. Matrix techniques for modelling ultrasonic waves in multilayered media. IEEE Transactions on Ultrasonics, Ferroelectrics and Frequency control, 42(4):525-542, 1995.

[4] J. C. P. McKeon and M. K. Hinders. Parallel projection and crosshole lamb wave contact scanning tomography. Journal of the Acoustical Society of America, 106(5):2568-2577, 1999.

[5] P. Fromme, P. D. Wilcox, M. J. S. Lowe, and P. Canvley. On the development and testing of a guided ultrasonic wave array for structural integrity monitoring. Ieee Transactions on Ultrasonics Ferroelectrics and Frequency Control, 53(4):777-785, 2006.

[6] D. Alleyne and P. Cawley. The long range detection of corrosion in pipes using lamb waves. In D.O. Thompson and D.E. Chimenti, editors, 
Review of Progress in Quantitative NDE, volume 14. Plenum Press, New York, 1994.

[7] J. O. Kim and H. H. Bau. On line, real-time densimeter - theory and optimsation. Journal of the Acoustical Society of America, 85:432 - 439, 1989.

[8] P. B. Nagy and R. M. Kent. Ultrasonic assessment of poisson's ratio in thin rods. Journal of the Acoustical Society of America, 98(5):269-2701, 1995.

[9] G. F. Miller and H. Pursey. The field and radiation impedance of mechanical radiators on the free surface of a semi-infinite isotropic solid. Proceedings of the Royal Society, 223:521-541, 1954.

[10] J.D. Achenbach. Wave propagation in elastic solids. North-Holland Publishing Company, 1975.

[11] M.J.S. Lowe and B.N. Pavlakovic. Disperse user manual, version 2.0.11d. Technical report, Imperial College of Science, Technology and Medicine, London, UK, 2001.

[12] R.D. Mindlin and E.A. Fox. Vibrations and waves in elastic bars of rectangular cross section. Journal of Applied Mechanics, pages 152-158, 1960.

[13] W. B. Fraser. Stress wave propagation in rectangular bars. International Journal of Solids and Structures, 5(4):379-397, 1969. 
[14] P. Wilcox, M. Evans, O. Diligent, M. Lowe, and P. Cawley. Dispersion and excitability of guided acoustic waves in isotropic beams with arbitrary cross section. In D. O. Thompson and D. E. Chimenti, editors, Review of Progress in quantitative NDE, volume 20, 2002.

[15] O. M. Mukdadi, Y. M. Desai, S. Datta, A. H. Shah, and A. J. Niklasson. Elastic guided waves in a layered plate with rectangular cross section. Journal of the Acoustical Society of America, 112(5):1766 - 1779, 2002.

[16] Hayashi T., Song W., and Rose J.L. Guided wave dispersion curves for a bar with an arbitrary cross-section, a rod and rail example. Ultrasonics, $41: 175-183,2003$.

[17] L. Gavric. Computation of propagative waves in free rail using a finite element technique. Journal of Sound and Vibration, 185(3):531-543, 1995.

[18] S. Finnveden. Evaluation of modal density and group velocity by a finite element method. Journal of Sound and Vibration, 273(1-2):51-75, 2004.

[19] M. V. Predoi, M. Castaings, B. Hosten, and C. Bacon. Wave propagation along transversely periodic structures. Journal of the Acoustical Society of America, 121(4):1935-1944, 2007.

[20] D. Hitchings. Fe77 user manual. Technical report, Imperial College of Science, Technology and Medicine, London, UK, 1994.

[21] ABAQUS Reference Manuals, Version 6.5. ABAQUS Inc., Rising Sun Mills, 166 Valley Street, Providence, RI 02909-2499, USA, 2004. 
[22] L. Moreau, M. Castaings, B. Hosten, and M. V. Predoi. An orthogonality relation-based technique for post-processing finite element predictions of waves scattering in solid waveguides. Journal of the Acoustical Society of America, 120(2):611-620, 2006.

[23] M. Drodz, L. Moreau, Castaings M., M.J.S. Lowe, and P. Cawley. Efficient finite element modelling of absorbing regions for boundaries of guided wave problems. In D. O. Thompson and D. E. Chimenti, editors, Review of Progress in Quantitative NDE, 2005.

[24] P. J. Torvik. Reflection of wave trains in semi-infinite plates. Journal of the Acoustical Society of America, 41:346 - 353, 1967.

[25] D. Alleyne and P. Cawley. A two-dimensional fourier transform method for the measurement of propagating multimode signals. Journal of the Acoustical Society of America, 89(3):1159-1168, 1991. 


\section{List of Figures}

1 Sketch a) of the strip geometry considered in this paper and b) of the geometry of the FE model used to obtain the dispersion curves of a strip of rectangular cross section (see text for dimensions). . . . . . . . . . . . . . 26

2 a) Sketch of the frequency-wavenumber results obtained from a FE eigen solver at different cyclic orders. b) Sketch of the frequency-wavenumber results transformed into the phase velocity frequency domain. . . . . . . . . . . . . . 27

3 Dispersion curves for a $1 \mathrm{~mm}$ thick and $30 \mathrm{~mm}$ wide rectangular steel strip determined by FEM a) frequency-wavenumber b) phase velocity frequency. Two interesting modes that correspond to the lowest order shear horizontal mode (SH0*) and the lowest order flexural mode(A0*) that is symmetric with respect to its width are highlighted by the bold dashed (- -) lines. For comparison c) and d) show the phase velocity dispersion curve of a 1 and $30 \mathrm{~mm}$ thick infinitely wide plate.

4 a)Phase velocity dispersion curves for a $1 \mathrm{~mm}$ thick steel strip of width $\left.30 \mathrm{~mm}(-), 15 \mathrm{~mm}(--)^{-}\right)$and $7.5 \mathrm{~mm}(-\cdots \cdot-\cdot)$. b)Phase velocity dispersion curves of the curves in a) but plotted against the frequency-width product. . . . . . . . . 29

5 Modulus of the displacement mode shapes of the SH0* mode of a $1 \mathrm{~mm}$ thick and $30 \mathrm{~mm}$ wide rectangular steel strip in the $\mathrm{x}, \mathrm{y}$ and $\mathrm{z}$ directions at different frequencies. . . . . . . . 30 
6 Evolution of the $\mathrm{SH}^{*}$ mode y displacement mode shape of a $30 \mathrm{~mm}$ wide and $1 \mathrm{~mm}$ thick steel strip over a range of frequencies $(200 \mathrm{kHz}$ to $3 \mathrm{MHz}$ in steps of $100 \mathrm{kHz}) \ldots \ldots . . . . . .31$

$7 \quad$ Sketch of the 2D plane stress model that was defined in ABAQUS to analyse the effect of different excitation force profiles. . . . 31

$8 \quad$ Y-direction displacement field output of the plane stress steady state frequency domain finite element model of a steel strip (15mm) under a) rectangular excitation force profile b) exact mode shape excitation force profile c) triangular excitation force profile across the width of the strip at $2 \mathrm{MHz}$. The difference between cases b) and c) is marginal and only visible at the strip edge. . . . . . . . . . . . . . . 32

9 Phase velocity dispersion curve of the A0 mode (-) of a $1 \mathrm{~mm}$ thick steel plate and of the $\mathrm{A} 0^{*}$ mode $\left(-\right.$ - $\left.^{-}\right)$of a $1 \mathrm{~mm}$ thick and 30mm wide steel strip. . . . . . . . . . . . . 33

10 Modulus of the displacement mode shape in the $\mathrm{x}, \mathrm{y}$ and $\mathrm{z}$ direction of the $\mathrm{A} 0^{*}$ mode in a $1 \mathrm{~mm}$ thick and $30 \mathrm{~mm}$ wide strip at different frequencies. . . . . . . . . . . . . . . . 34

11 Evolution of the $\mathrm{A}^{*}$ mode $\mathrm{x}$ displacement mode shape of a $30 \mathrm{~mm}$ wide and $1 \mathrm{~mm}$ thick steel strip over a range of frequencies $(500 \mathrm{kHz}$ to $2.5 \mathrm{MHz}$ in steps of $250 \mathrm{kHz}) . \ldots . . . . .35$

$122 \mathrm{MHz}$ centre frequency $\mathrm{SH}{ }^{*}$ signal received in pulse echo mode from a standard shear transducer coupled to the end of a $15 \mathrm{~mm}$ wide, $1 \mathrm{~mm}$ thick and $300 \mathrm{~mm}$ long stainless steel strip. 36 
13 a) Sketch of the in-plane laser doppler vibrometer scanning configuration along the strip. b) Two dimensional Fourier transform of in-plane surface displacements (polarised in the width direction of the strip) along the centre line of the $1 \mathrm{~mm}$ thick and 30mm wide steel strip. The dashed line (- - -) shows the predicted dispersion relation for the $\mathrm{SH}^{*}$ mode of steel $\left(\rho=7932 \mathrm{~kg} / \mathrm{m}^{3}, \mathrm{Cl}=6000 \mathrm{~m} / \mathrm{s}, C s=3060 \mathrm{~m} / \mathrm{s}\right) . \ldots 37$

14 a) Pulse echo signal received from a $30 \mathrm{~mm}$ wide and $0.2 \mathrm{~mm}$ thick stainless steel strip using a 10 cycle Hanning windowed toneburst with $2 \mathrm{MHz}$ centre frequency. b) Spectrum of the signal in a) . . . . . . . . . . . . . . . . . 38

15 a) Sketch of the out-of-plane laser doppler vibrometer measurements on a strip. b) Two dimensional Fourier Transform of the out-of-plane displacements of the centre line of a $0.2 \mathrm{~mm}$ thick and $30 \mathrm{~mm}$ wide steel strip along the centre line of the strip. The dashed line (- - -) shows the predicted dispersion relation for the $A 0^{*}$ mode of steel $\left(\rho=7932 \mathrm{~kg} / \mathrm{m}^{3}, \mathrm{Cl}=6000\right.$ $\mathrm{m} / \mathrm{s}, C s=2840 \mathrm{~m} / \mathrm{s}) . \ldots \ldots \ldots \ldots$ 

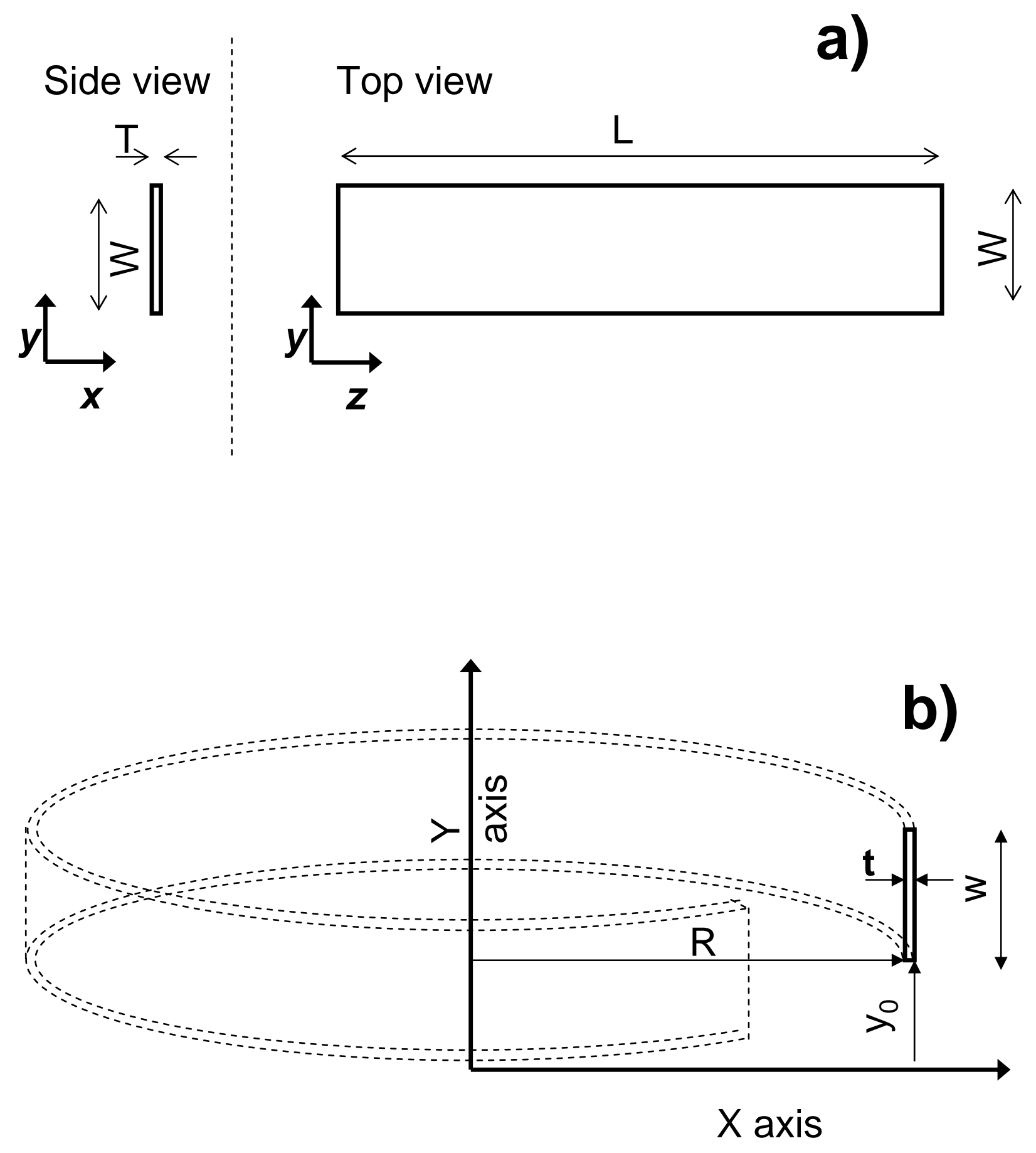
Discrete Frequency

solutions

a)

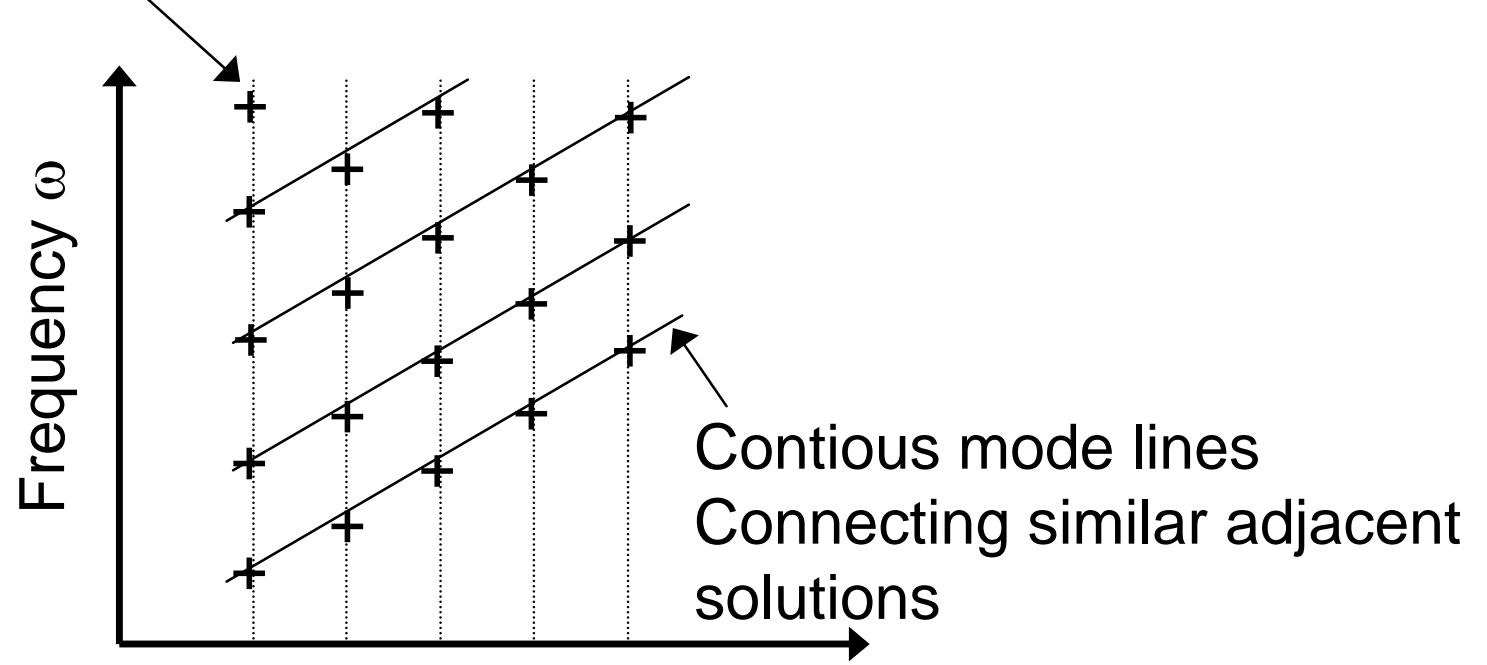

$\begin{array}{lllllll}k_{1} & k_{2} & k_{3} & k_{4} & k_{5} & \ldots\end{array}$

Discrete Wavenumbers

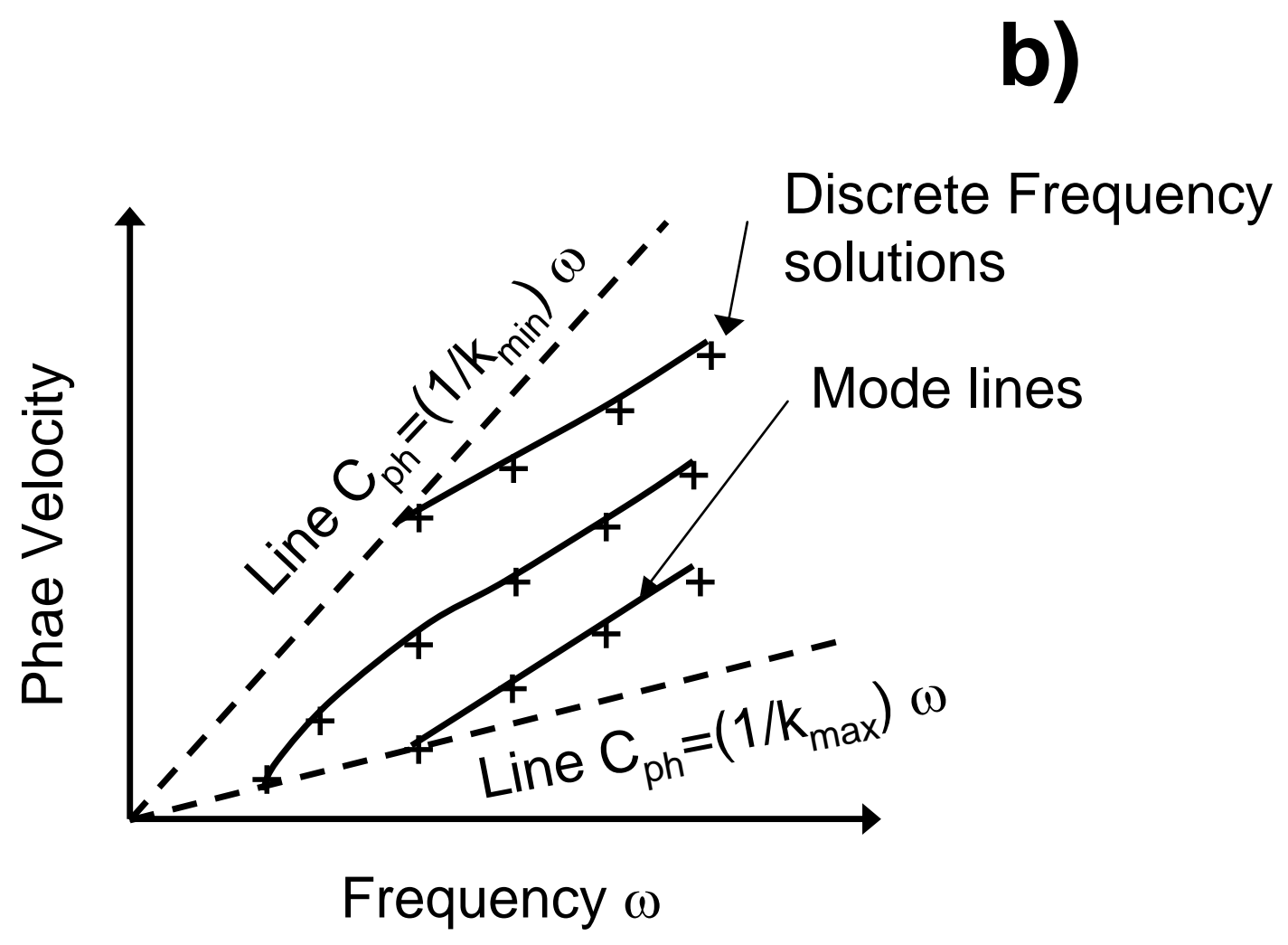



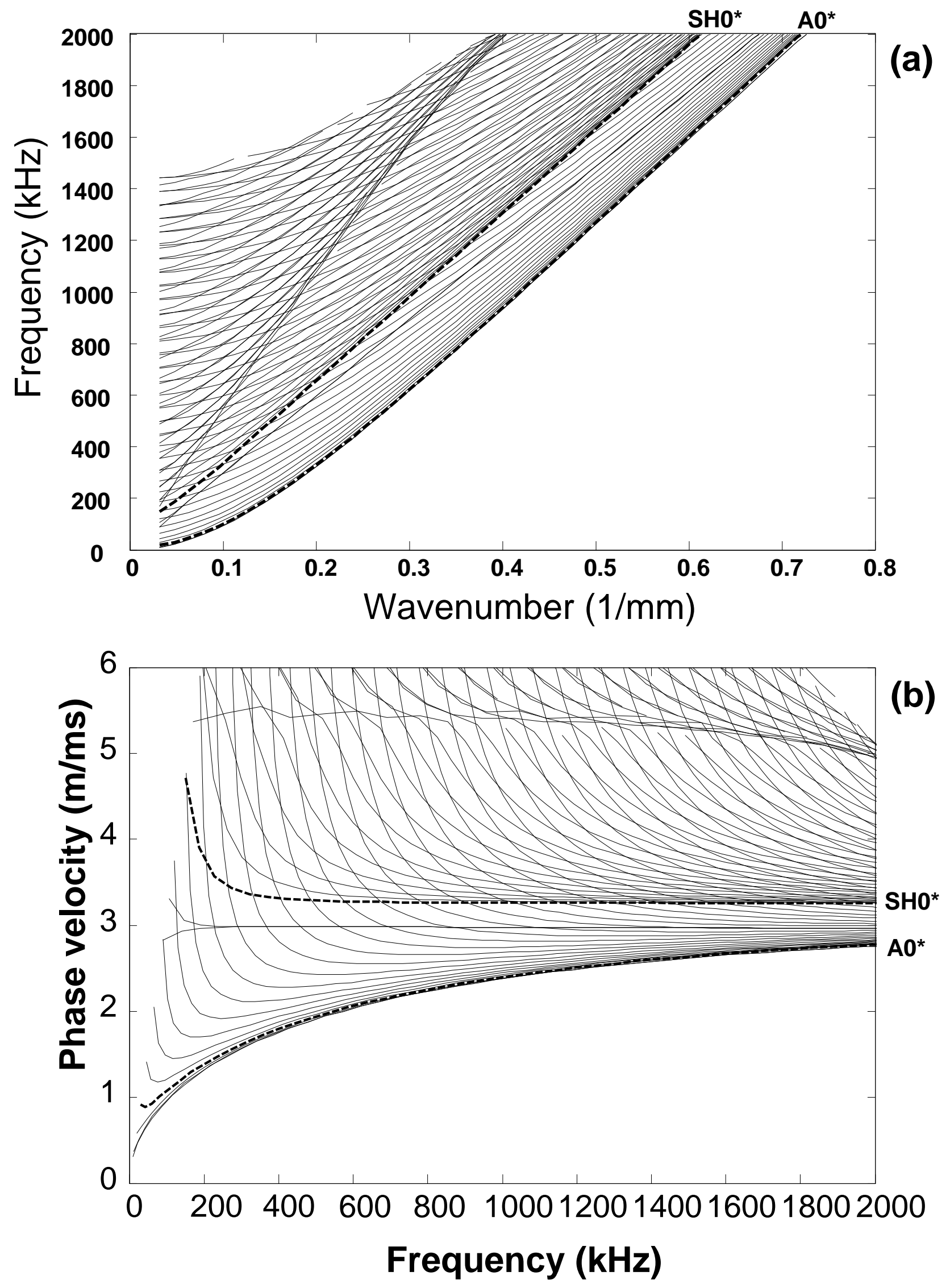


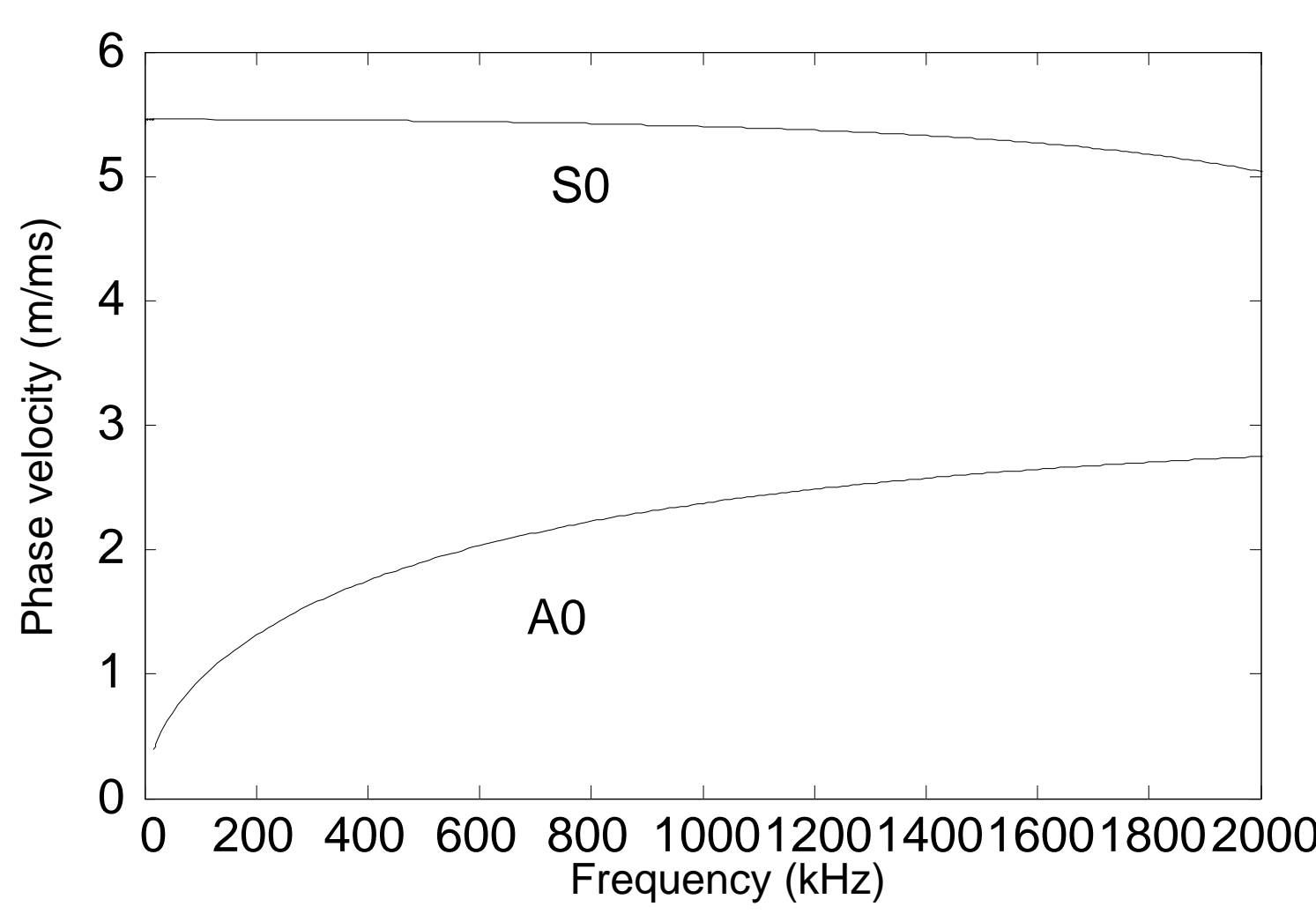

c)

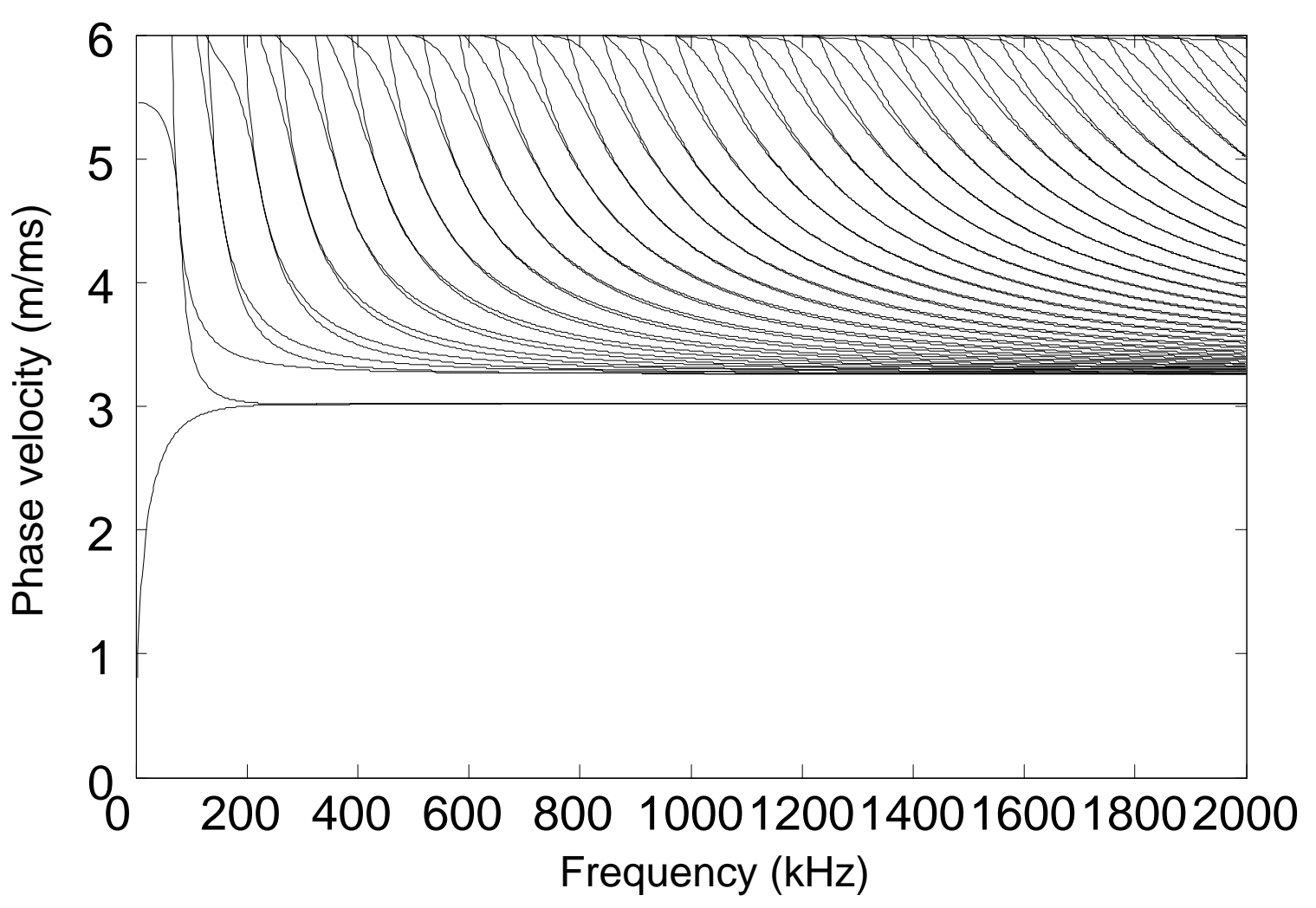

d) 


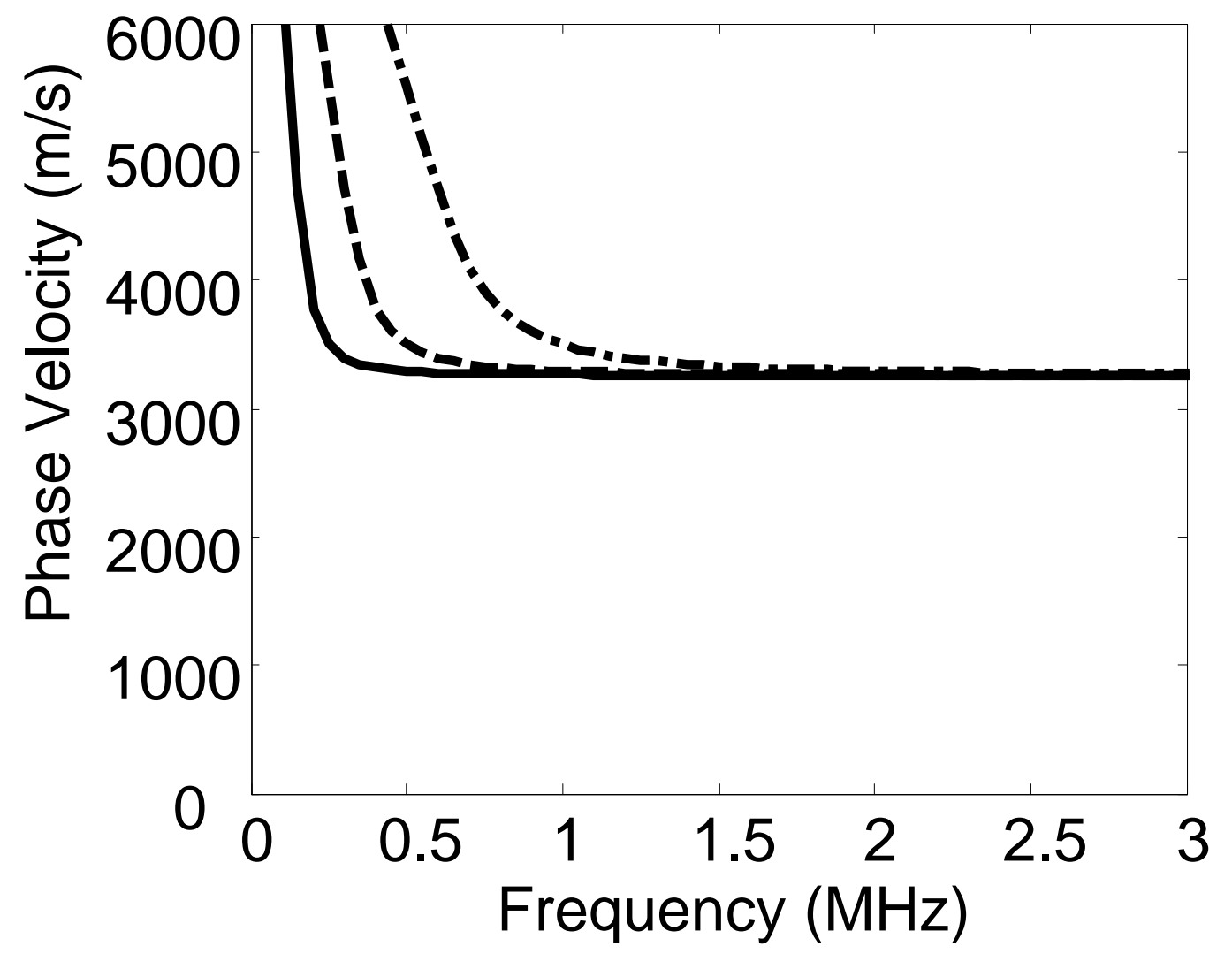

a)

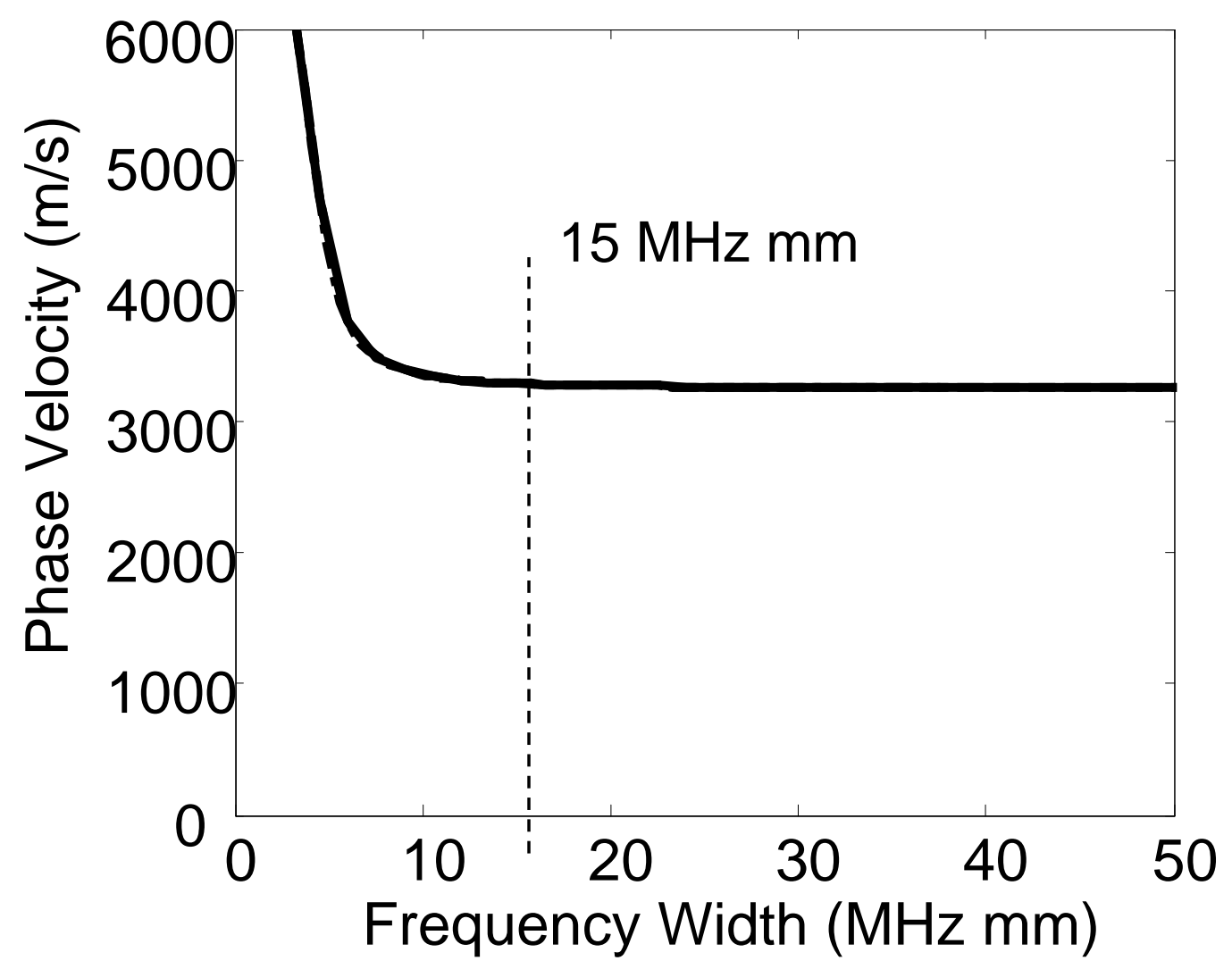

b) 


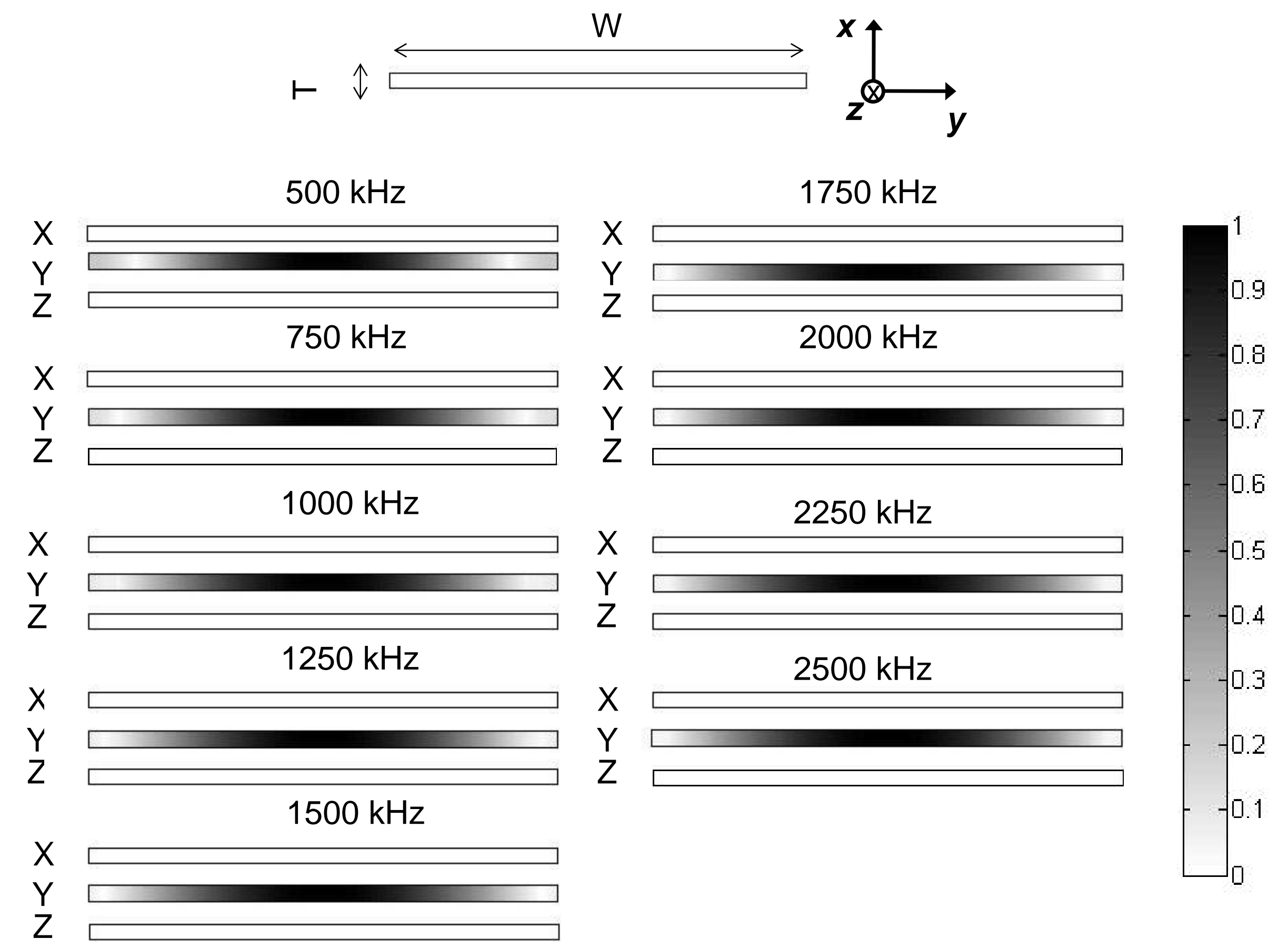




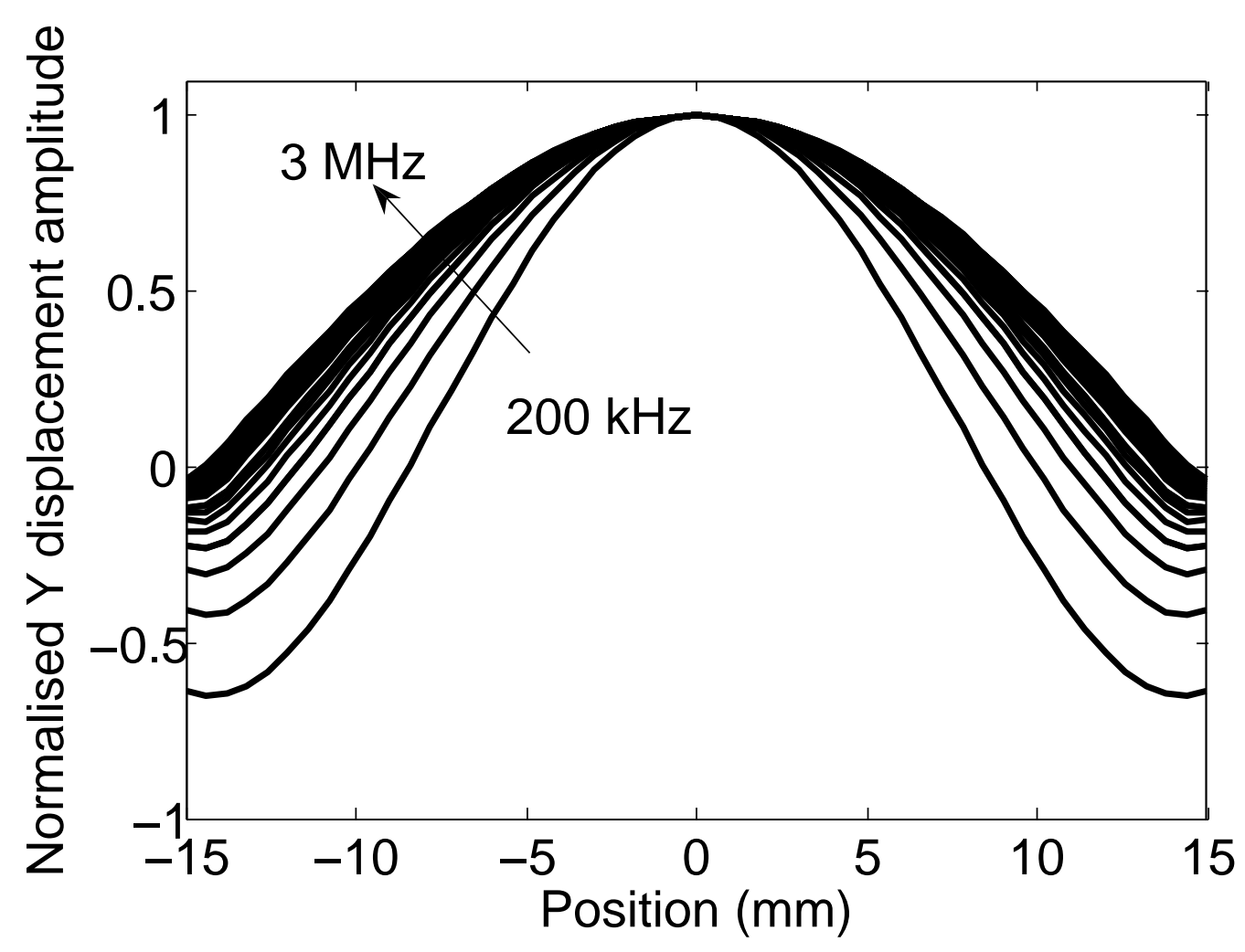


Excitation Absorbing Region

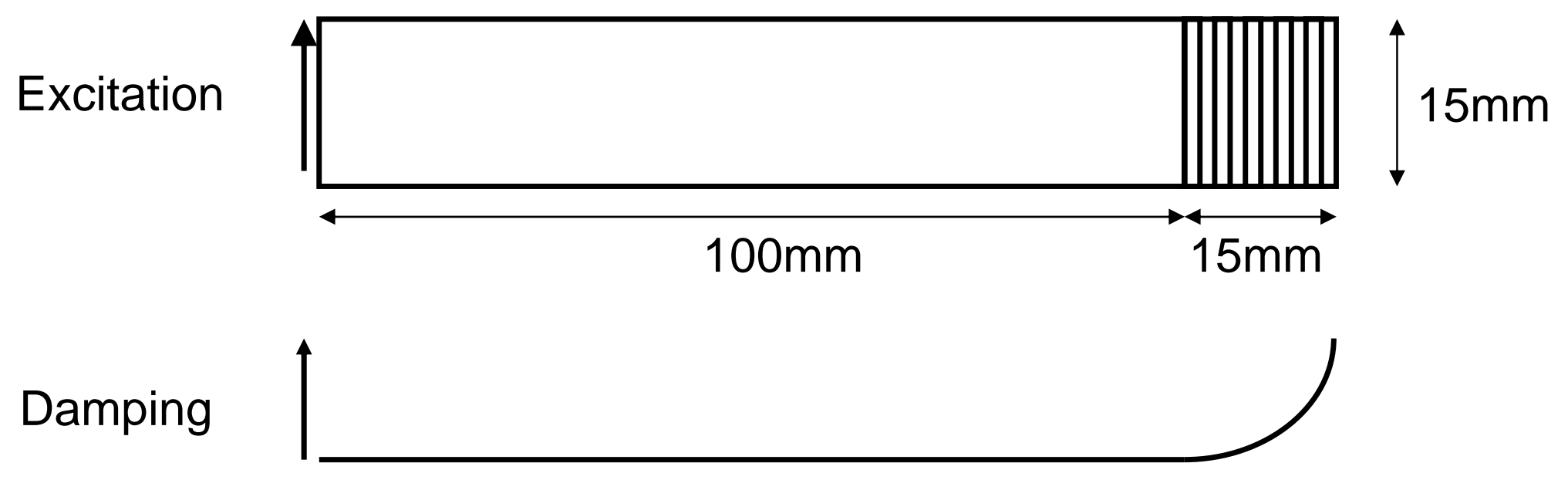


Excitation Force Profile Strip Y-Displacement Field

a)
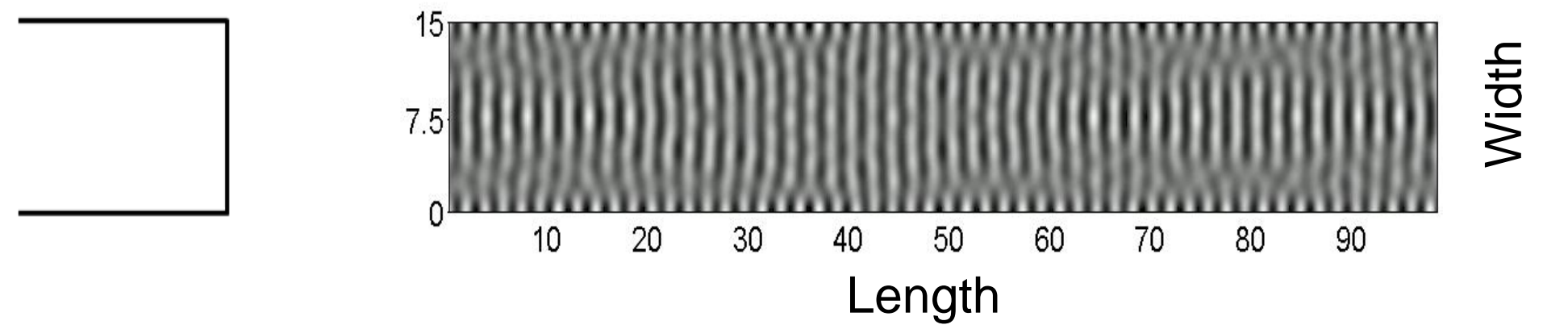

b)
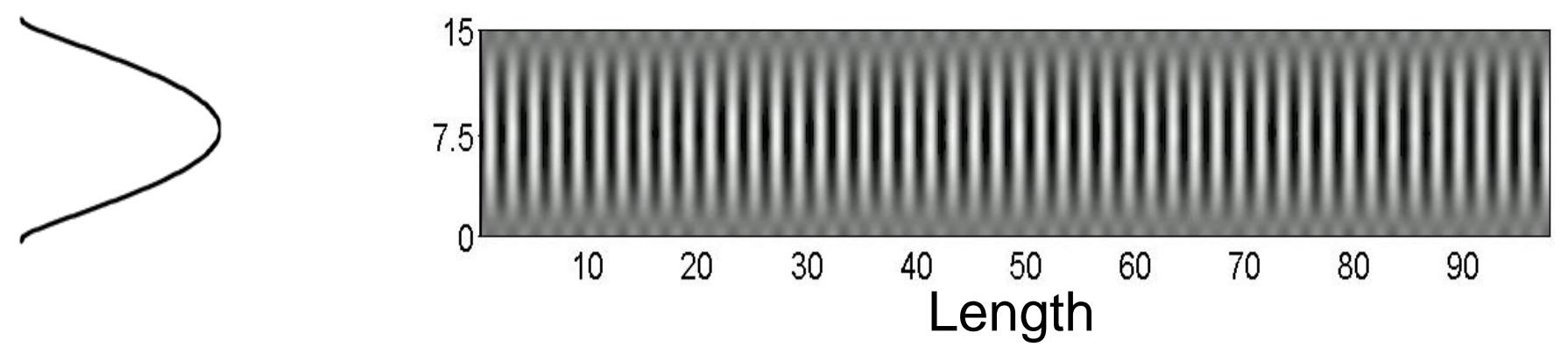

c)
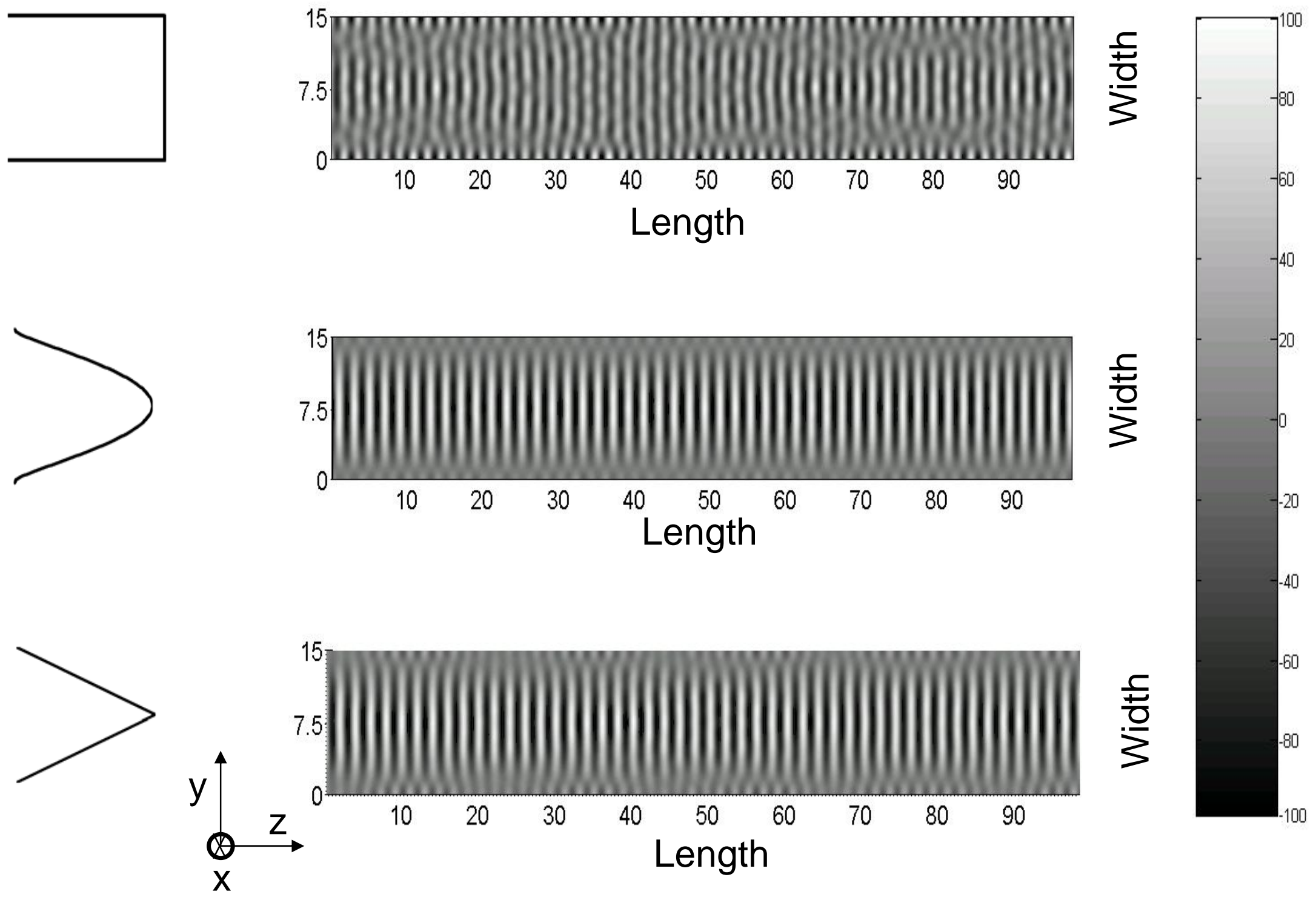


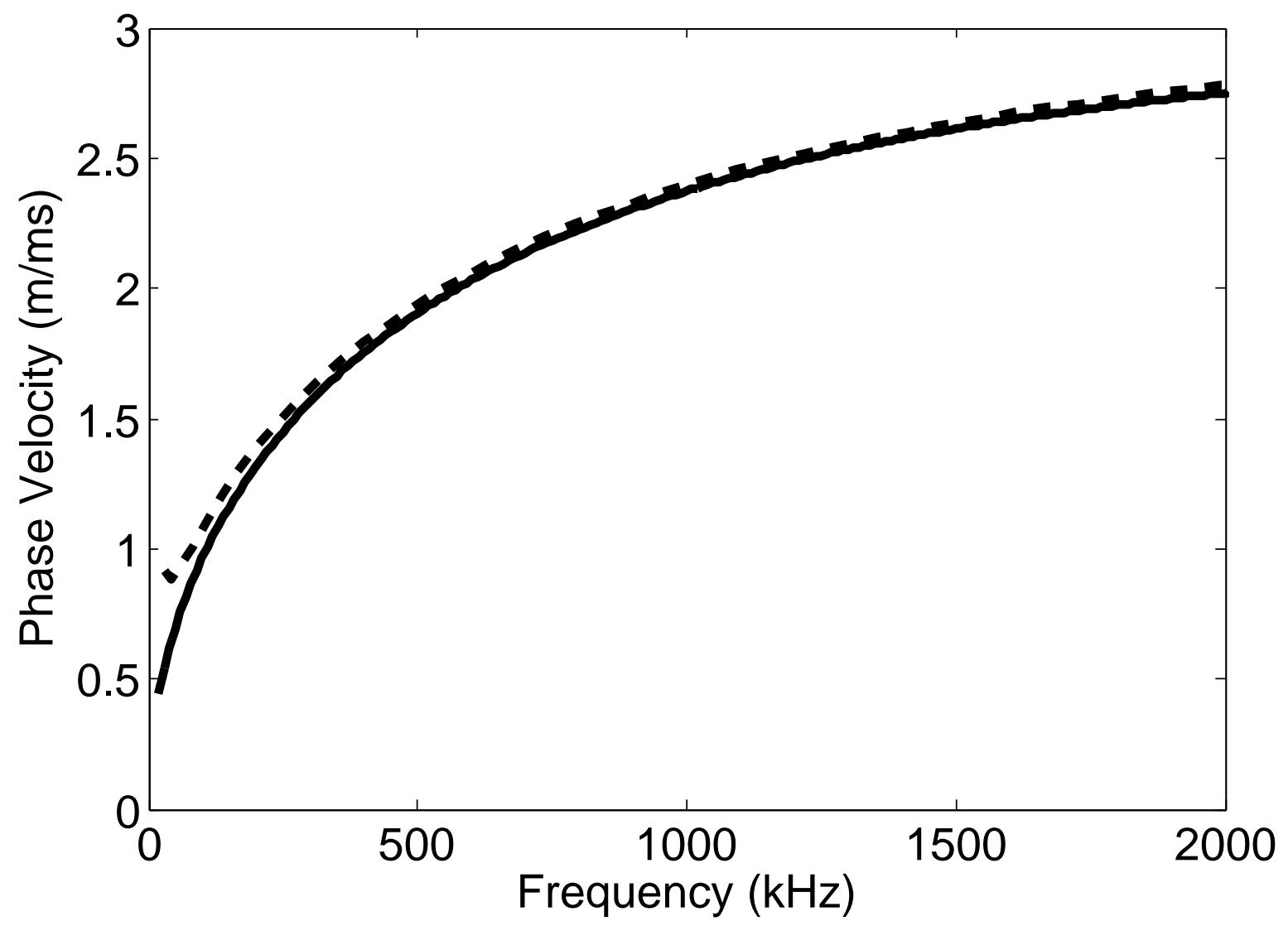




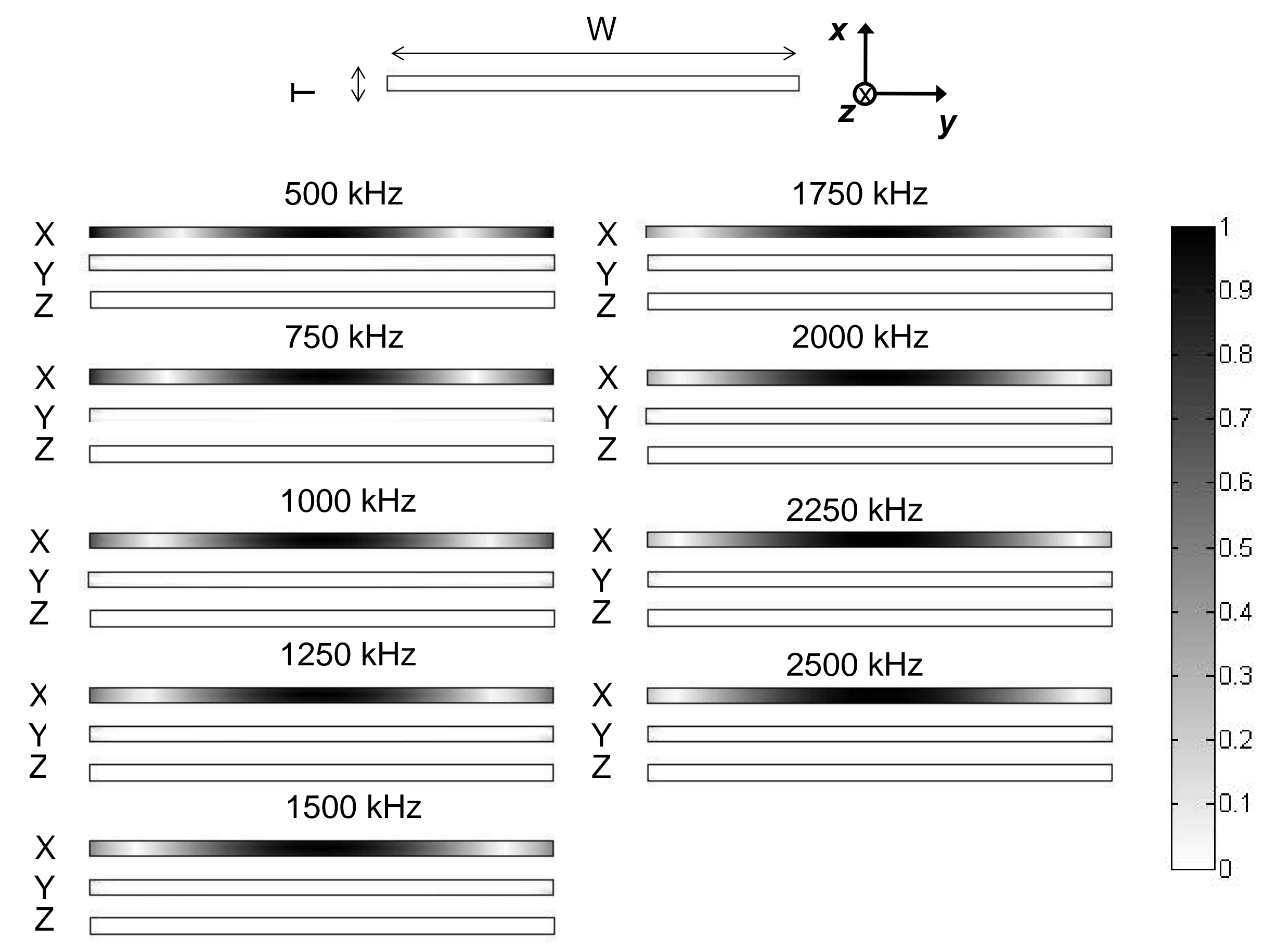




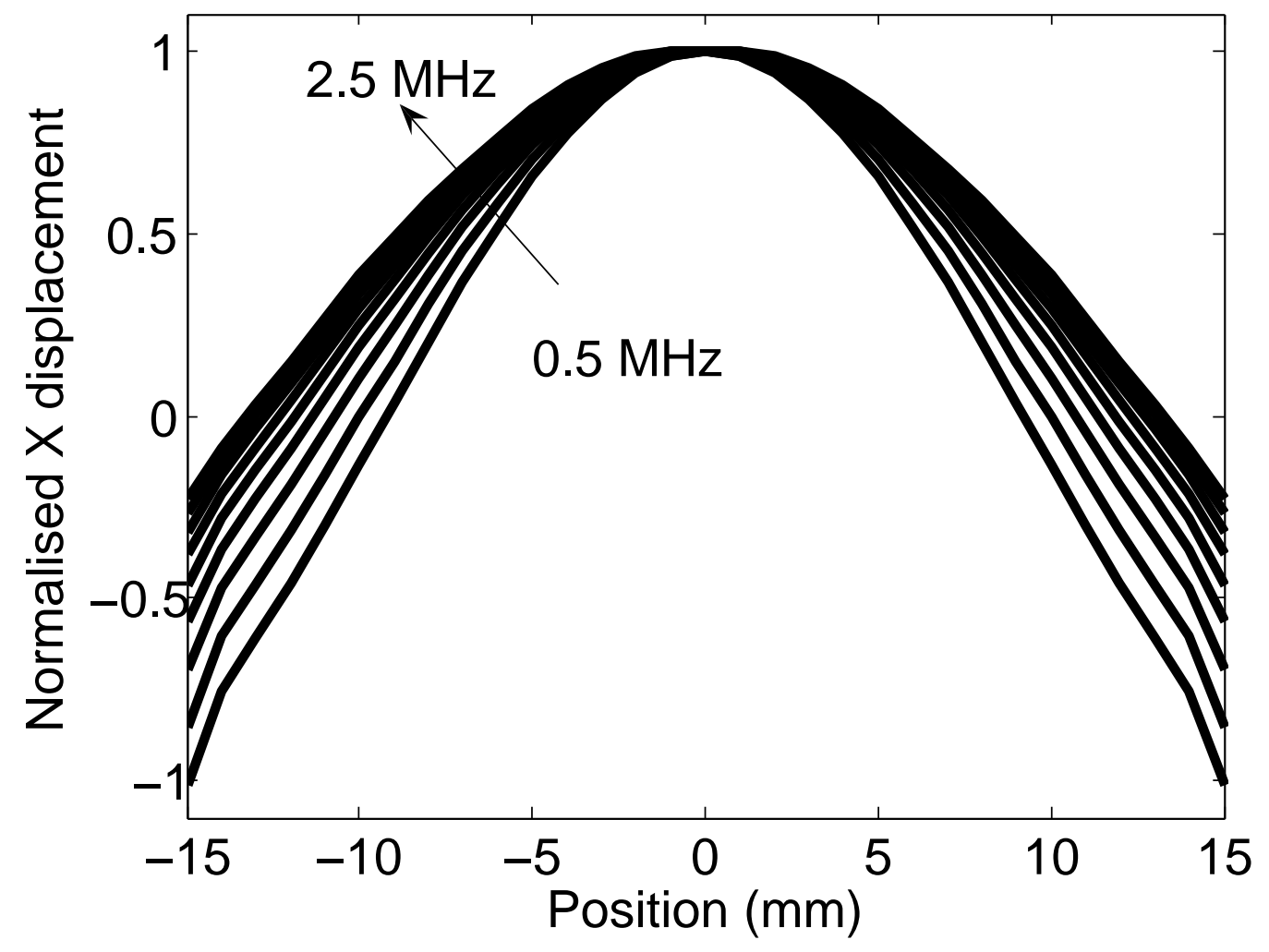




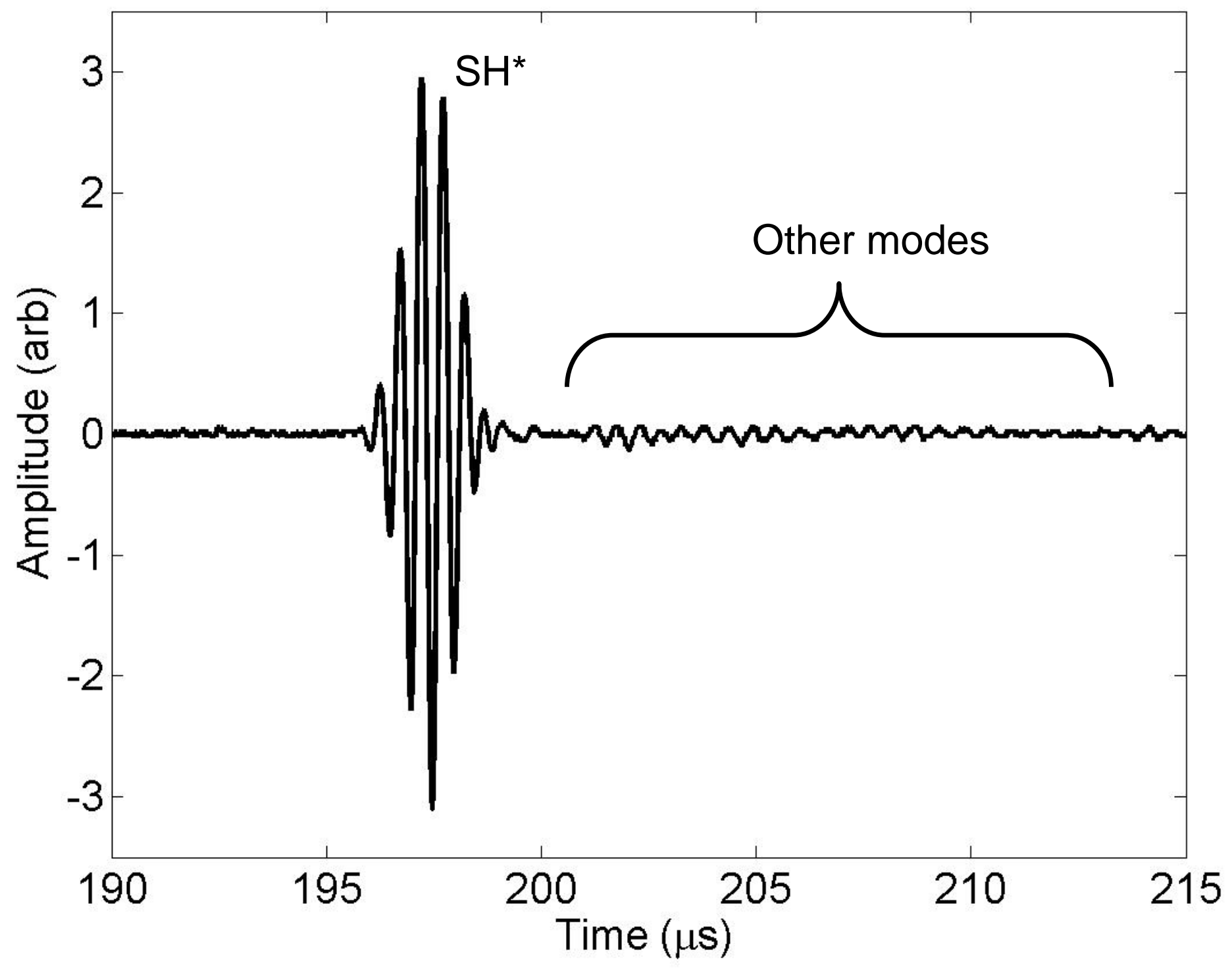




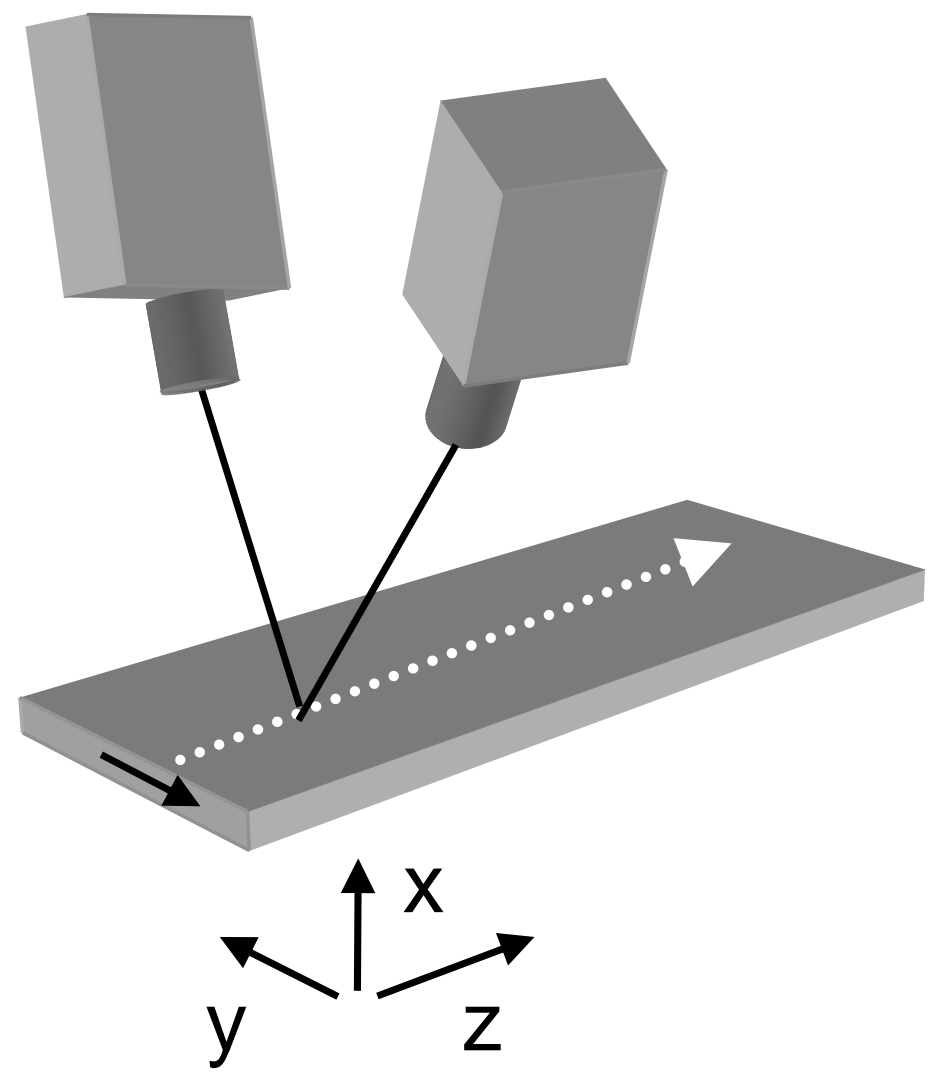

a)

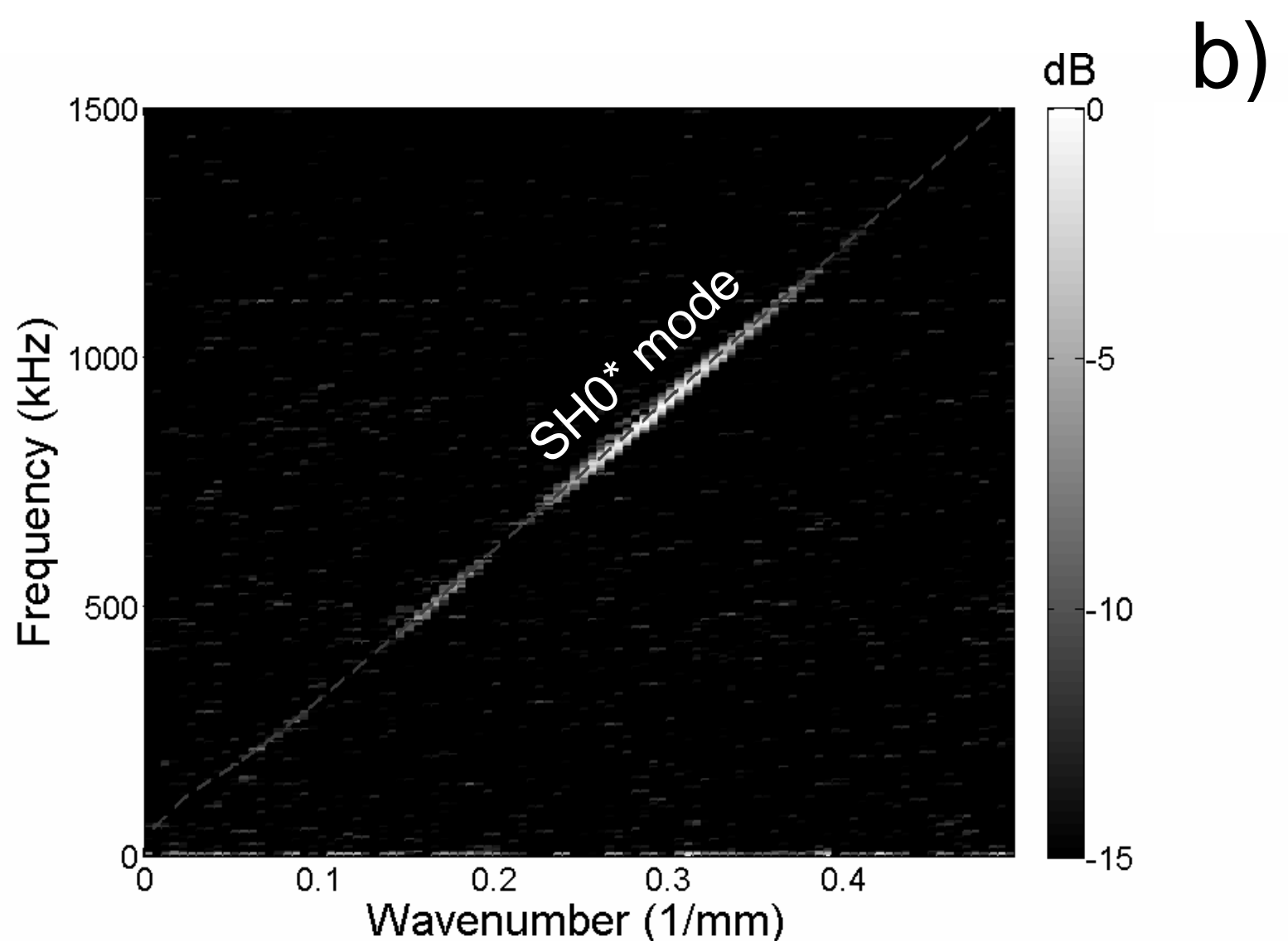



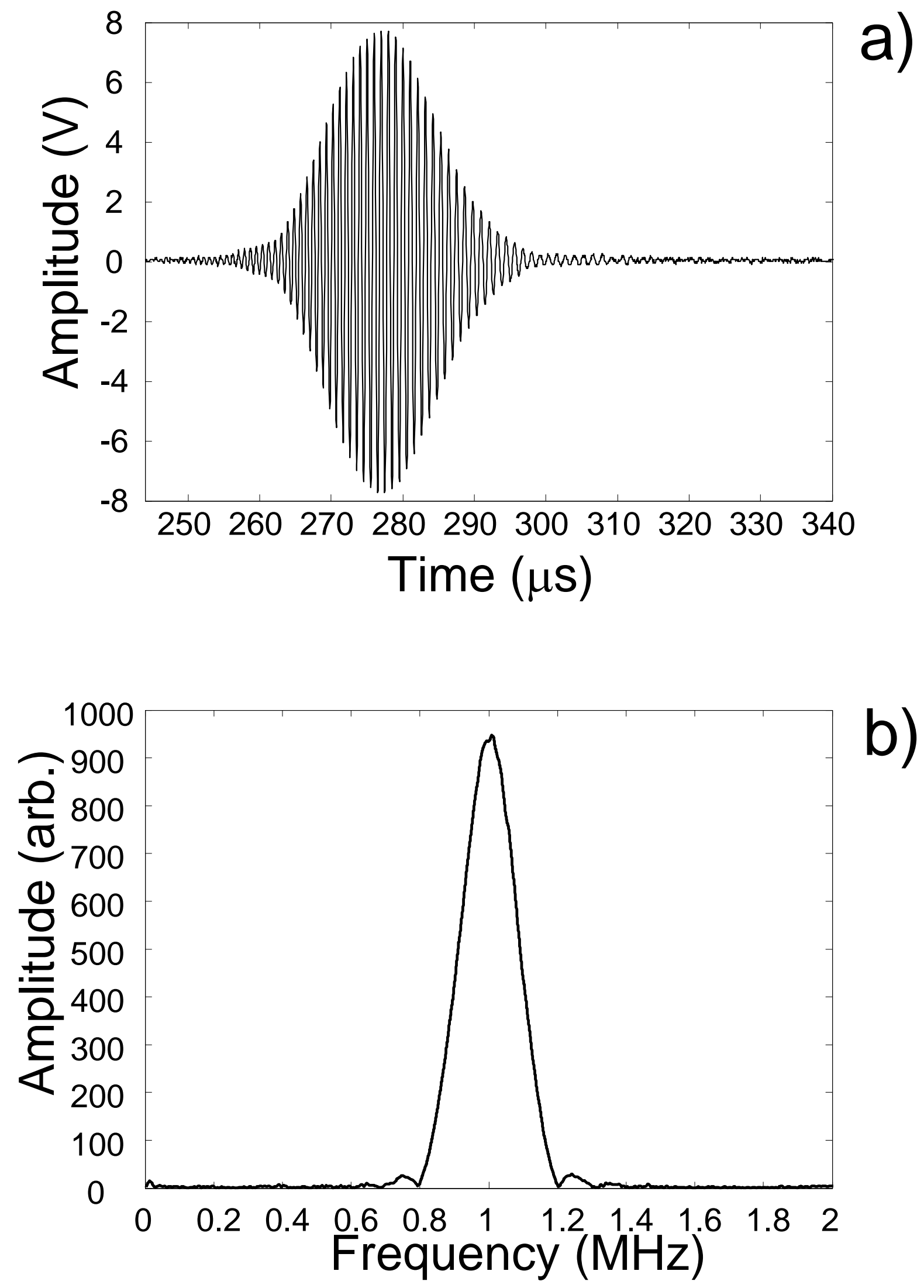


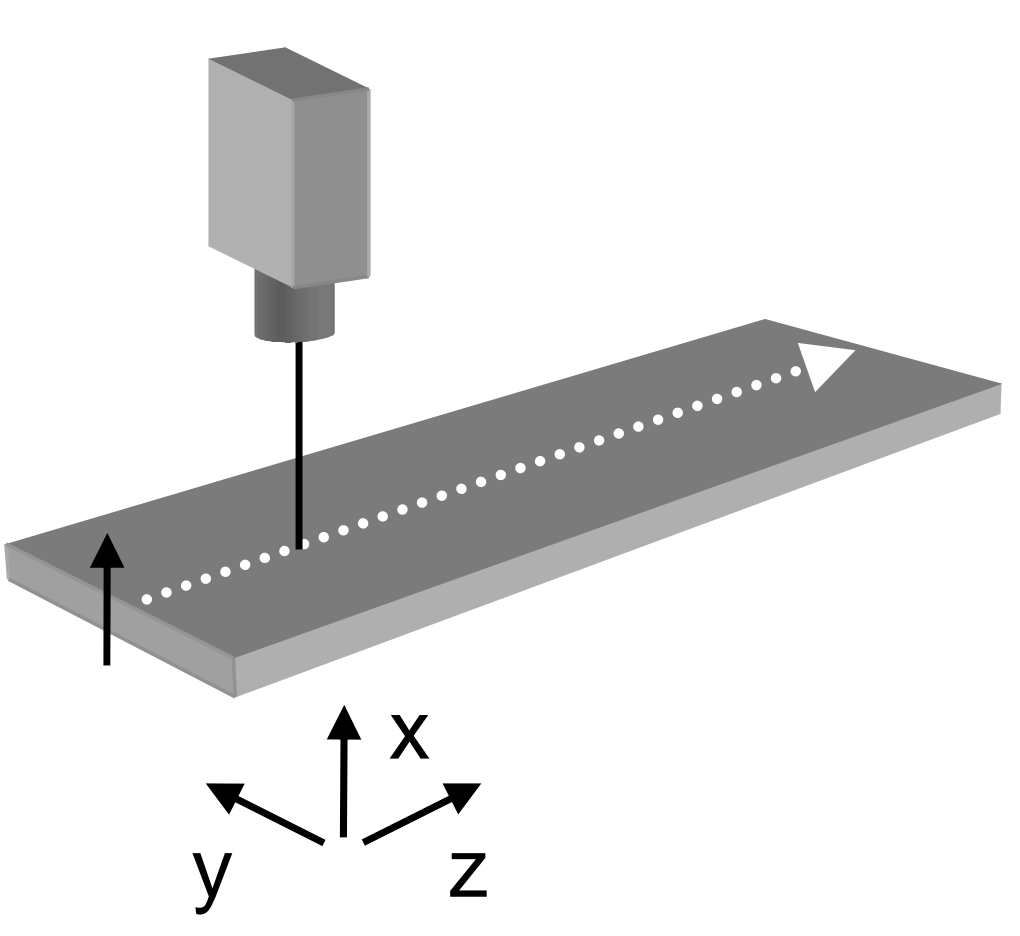

a)

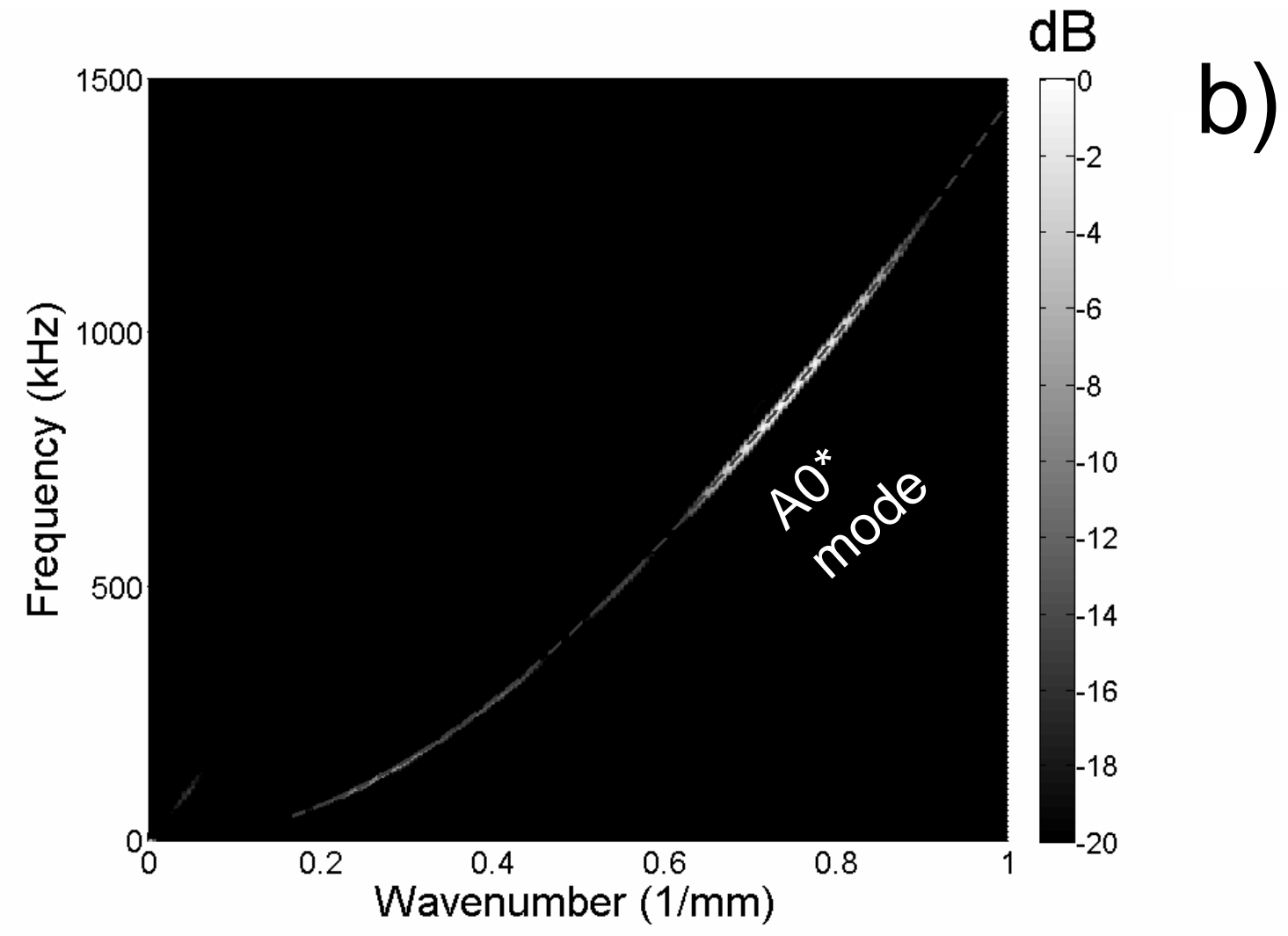

